\title{
Stochastic Thermodynamics of Nonlinear Electronic Circuits: A Realistic Framework for Computing Around $k T$
}

\author{
Nahuel Freitas $\odot,{ }^{1}$ Jean-Charles Delvenne, ${ }^{2}$ and Massimiliano Esposito ${ }^{1}$ \\ ${ }^{1}$ Complex Systems and Statistical Mechanics, Department of Physics and Materials Science, \\ University of Luxembourg, L-1511 Luxembourg, Luxembourg \\ ${ }^{2}$ Institute of Information and Communication Technologies, Electronics and Applied Mathematics, \\ Université catholique de Louvain, Louvain-La-Neuve, Belgium
}

(Received 1 October 2020; revised 2 July 2021; accepted 8 July 2021; published 22 September 2021)

\begin{abstract}
We provide a general theory of nonlinear electronic circuits subjected to thermal noise. The devices constituting the circuit can have arbitrary $I-V$ curves but must display shot noise. This theory includes tunnel junctions, diodes, and MOS transistors in subthreshold operation, among others. The stochastic nonequilibrium thermodynamics of these circuits is also established. The irreversible entropy production is expressed in terms of thermodynamic potentials and forces, and its fluctuations satisfy fluctuation theorems. Our theory is ideally suited to formulate a thermodynamics of computing with realistic architectures, where the reduction in transistor size and operating voltages make thermal fluctuations increasingly important. We demonstrate this point in two ways: first, by proposing a stochastic model of a CMOS inverter whose actual transfer function deviates from the deterministic one due to nonequilibrium fluctuations, and, second, by proposing a low-power full-CMOS design for a probabilistic bit (or binary stochastic neuron) exploiting intrinsic noise.
\end{abstract}

DOI: $10.1103 /$ PhysRevX.11.031064

Subject Areas: Complex Systems, Electronics

Statistical Physics

\section{INTRODUCTION}

The growing energy consumption of data-intensive technologies is raising concern [1,2]. Semiconductor-based electronic circuits constitute the dominant architecture implementing the information processing underlying these technologies. In order to reduce energy consumption, these circuits need to operate in regimes where the informationcarrying signals are increasingly close to thermal fluctuations [3-9]. Proposals have, for instance, been made to trade reliability for energetic costs in certain applications [10-12] or to exploit intrinsic thermal fluctuations (instead of generating them at much higher energy cost with random number generators) when solving stochastic optimization problems [13-15]. But, despite that, a proper description of thermal noise in nonlinear electronic circuits remains an open problem, especially if one aims at preserving thermodynamic consistency [16-22]. Traditional methods employed in engineering are usually based on the linearization of the response of a given element around an operating point or consider only external noise generated

Published by the American Physical Society under the terms of the Creative Commons Attribution 4.0 International license. Further distribution of this work must maintain attribution to the author(s) and the published article's title, journal citation, and DOI. by linear resistors (the internal or intrinsic noise is first mapped to external sources) [19,23]. Although these approaches might offer accurate estimations of the noise in some applications, they are not thermodynamically consistent and are, therefore, not suited for situations where thermal fluctuations are relevant or, even more so, are exploited as a resource.

The discovery of the so-called fluctuation theorems [24-28] and the ensuing development of the theory of stochastic thermodynamics [27,29-31] established universal constraints on the thermal fluctuations of any system, even if it is highly nonlinear and arbitrarily away from thermal equilibrium. The central ingredient introducing thermodynamic consistency in stochastic thermodynamics are the local detailed balance (LDB) conditions, which relate the log-ratio between the forward and backward rates of a given fluctuation or transition to the corresponding entropy production in the environment. In this way, the LDB conditions connect the dynamical description to the thermodynamics, even far from equilibrium. The theory is used to study colloidal particles, chemical reaction networks, molecular motors [27,32-34], and also electronic systems. It is applied to linear electrical circuits ranging from simple circuits [35-37] to complex networks (even in quantum regimes) [38], but these circuits are of limited use to implement computations. Following experimental and theoretical progress on the study of nonlinear 
single-electron devices and Coulomb blockade systems [16,39-43], stochastic thermodynamics was also used to reach a detailed understanding of thermal fluctuations in these systems [44-51]. The nature of the conduction channels (typically tunnel junctions) and the nanoscopic size of the conductors (implying low capacitances and high charging energies) allow one to design circuits that process information with very low energy requirements [40]. The logical states here are represented by the presence of just one or a few electrons [52]. Unfortunately, the need to operate at low temperatures and challenges in the fabrication of these devices have so far prevented their practical application in computing. In the foreseeable future, regular complementary metal-oxide-semiconductor (CMOS) circuits will probably remain the most relevant platform for computing [53,54]. In this context, the quest for speed and low energy consumption fueled spectacular progress in the miniaturization of transistors, and nowadays integrated circuits with a typical feature size of around $5 \mathrm{~nm}$ can be mass produced. In these circuits, a transistor can be activated by just a few hundred electrons in its gate terminal. Thus, CMOS circuits are approaching regimes where a description in terms of single electrons becomes necessary [9]. The fact that both single-electron devices and CMOS transistors (in some modes of operation) display shot noise [55] suggests that the rigorous tools and methods that have been used to model and simulate single-electron devices could also be applied to study CMOS circuits. While recent studies use stochastic thermodynamics for the detailed characterization of individual devices such as diodes and transistors [51,56,57], the study of complex networks and circuits comprising such devices remains unexplored.

Stochastic thermodynamics also offers a framework to study the energetic costs of computation in a systematic way $[58,59]$. Although it was realized early on that information processing can, in principle, be done without energy expenditure $[60,61]$, this fact is true only in idealized setups where either the computation is extremely slow or the computing device is perfectly isolated from the environment. However, real computations are done in finite time and in noisy environments and, thus, dissipate energy. Stochastic thermodynamics has been used to resolve contentious issues in thermodynamics of computation such as the relation between thermodynamic and logical reversibility [58,59,62], the energetic costs associated with measurement and erasure [62-65], and the cyclic operation of computing devices [58]. It is also used to evaluate the dissipation of finite-time processes [66-69] and to design optimal erasing protocols [70-73]. The thermodynamic costs associated with the structure of complex information processing networks are also studied [74,75]. However, these efforts involve either extremely simple and idealized models or abstract formulations aimed at obtaining fundamental bounds, where no particular computing architecture is considered.
In this paper, we report three major achievements. First, in Secs. II-IV, we develop a general formalism to construct thermodynamically consistent stochastic dynamical descriptions of arbitrary circuits made of devices displaying shot noise. In this way, we resolve the longstanding problem of rigorously describing thermal noise in nonlinear electronic circuits by providing an alternative to the usual methods employed in engineering, which are based on linear response around an operating point. To do so, we combine the LDB conditions with the $I-V$ curve characterization of a given device, in order to describe its stochastic behavior when it is introduced in an arbitrary circuit. This procedure fully captures the charging effects resulting from electromagnetic rearrangements in the circuit when electron transfers occur [39]. Neglecting those charging effects compromises thermodynamic consistency and leads to unphysical results (e.g., perpetuum mobile). Our formalism can seamlessly accommodate many different devices like tunnel junctions [40,47], diodes [20], MOS transistors in subthreshold operation [20,55,76], or more exotic devices like nanoscale vacuum channel transistors [77]. The devices may even be time-dependently driven and at different temperatures.

Second, because the dynamical description is by construction thermodynamically consistent (i.e., compatible with the LDB conditions), we can employ stochastic thermodynamics for a complete thermodynamic characterization of these circuits. This characterization is done in Secs. V and VI, where the first and second laws of thermodynamics are formulated at the level of the ensemble-averaged description as well as along single stochastic trajectories. We also establish the relation between heat and electric currents and identify the thermodynamic potentials and forces at work in these circuits. We finish by formulating a general version of the Landauer principle as well as the different fluctuation theorems known to date.

Third, by formulating the stochastic thermodynamics of a large family of technological relevant electronic circuits, we provide a rigorous framework to study thermodynamics of computation implemented with realistic architectures instead of toy models. To substantiate this claim, in Sec. VII, we construct and analyze a stochastic model of a CMOS inverter (or NOT gate) and of a probabilistic bit ( $p$-bit). The CMOS inverter is an important primitive in electronic design, from which more complex devices like oscillators and memories can be built. We show how, due to nonequilibrium fluctuations, the transfer function of the gate deviates from the one obtained by a deterministic treatment. We also compute the full counting statistics of the current fluctuations and illustrate the validity of a detailed fluctuation theorem. The $p$-bit can be considered as a faulty memory, with a controllable bias and error rate. They are a physical implementation of what in machine learning is known as a binary stochastic neuron [78,79]. Such devices were recently employed in proof-of-concept 
experiments to solve stochastic optimization problems and emulate artificial neural networks [13,14]. Other proposals to exploit physical noise include cryptographic applications [80]. To the best of our knowledge, our design is the first full-CMOS proposal for a $p$-bit exploiting intrinsic noise and can be implemented with current technology. The methods employed in these examples can be directly applied to model arbitrary logic gates at the stochastic level, in both asynchronous computing schemes or synchronous ones requiring an external clock signal.

Our work provides bridges between computer engineering, mesoscopic physics, and nonequilibrium statistical physics. In doing so, it may contribute to the search for new practical and energy-efficient computing paradigms and also to the design of experiments taking advantage of the versatility of electronic circuits in order to test new developments in statistical physics.

Please note that the reader mainly interested in the stochastic modeling of circuits might want to skip Secs. V and VI on a first read and go directly to the applications discussed in Sec. VII.

\section{BASIC SETUP}

We consider an arrangement of $N_{0}$ ideal conductors characterized by their total charge $\left\{q_{n}\right\}_{n=1, \ldots, N_{0}}$ and electrostatic potential $\left\{V_{n}\right\}_{n=1, \ldots, N_{0}}$, where the latter are measured with respect to some reference or "ground" (see Fig. 1). Basic electrostatic theory shows that the charges and potentials are related by the linear relation:

$$
q_{0}=C_{0} V_{0},
$$

where $\boldsymbol{q}_{\mathbf{0}}=\left(q_{1}, \ldots, q_{N_{0}}\right)^{T}$ and $\boldsymbol{V}_{\mathbf{0}}=\left(V_{1}, \ldots, V_{N_{0}}\right)^{T}$ are column vectors containing the charges and voltages, respectively, and the $N_{0} \times N_{0}$ symmetric matrix $\boldsymbol{C}_{\mathbf{0}}$ (known as the Maxwell capacitance matrix) encodes the mutual and

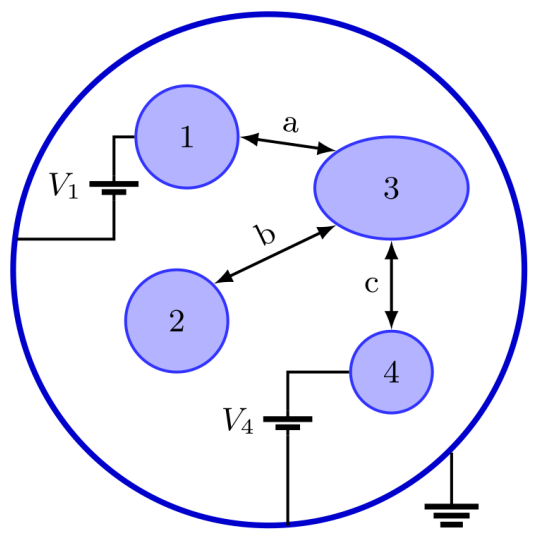

FIG. 1. A system of conductors. Two of them (1 and 4) are maintained at fixed potentials $V_{1}$ and $V_{4}$ by voltage sources. Elementary charges $q_{e}$ are interchanged between them by devices $a, b$, and $c$. self-capacitances of the conductors. These capacitances depend on the shape, size, and relative position and orientation of the conductors. The electrostatic energy contained in such a system is given by the quadratic form

$$
E=\frac{1}{2} q_{0}^{T} V_{0}=\frac{1}{2} V_{0}^{T} C_{0} V_{0}=\frac{1}{2} q_{0}^{T} C_{0}^{-1} q_{0} .
$$

Some of the conductors can have their potential fixed by voltage sources, and in that case we say that the circuit is open. We refer to conductors with fixed potentials as regulated conductors and to the rest as free conductors. Thus, we have $N_{0}=N+N_{r}$, where $N$ is the number of free conductors and $N_{r}$ the number of regulated conductors. The vector $\boldsymbol{q}_{\mathbf{0}}$ is split into vectors $\boldsymbol{q}$ and $\boldsymbol{q}_{r}$ containing the charges of the free and regulated conductors, respectively. If the circuit is open, then the degrees of freedom of the system are reduced. This result can be seen from Eq. (1), since fixing the potential of a conductor imposes a linear relationship between all the charges. Then, the state of the system is fully specified by the charges $\boldsymbol{q}$ of all the free conductors.

We also consider two-terminal devices or channels that allow the transport of elementary charges of value $q_{e}$ between pairs of conductors, forming a network or circuit. Each of these channels is modeled as a bidirectional Poisson process (BPP). This choice offers some generality while keeping the formalism concrete and simple and, as mentioned before, allows one to describe relevant devices like tunnel junctions [40,47], diodes [20], MOS transistors in subthreshold operation [55,76], and other devices like nanoscale vacuum channel transistors [77]. The common feature of all these devices is that they display shot noise (i.e., the current noise spectral density is proportional to the average current for large biases [81]; see Sec. III A). This feature is also a limitation of the BPP modeling choice, since it does not allow one to properly describe regular resistors or source-drain conduction in MOS transistors operating in saturation mode, where the current noise spectral density is approximately independent of the average current (as for Johnson-Nyquist noise) [81]. However, this limitation is not serious, especially if we are interested in the regime of ultralow energy consumption, where the subthreshold and unsaturated operation of MOS transistors is optimal [76].

Let $\rho=1, \ldots, M$ index the two-terminal devices present in the circuit. Then, given a device $\rho$ connecting conductor $n$ and $m$, we associate to it two basic Poisson processes: a "forward" one, in which an elementary charge is transported from conductor $n$ to conductor $m$, and the "reverse" one, in which a charge is transported in the opposite direction, with respective rates $\lambda_{\rho}(\boldsymbol{q}, t)$ and $\lambda_{-\rho}(\boldsymbol{q}, t)$. The forward direction is, of course, chosen arbitrarily. Note that the rates $\lambda_{ \pm \rho}(\boldsymbol{q}, t)$ depend explicitly on the full state $\boldsymbol{q}$ of the system, which allows one to model externally controlled 
conduction channels. Thus, in a transition $\pm \rho$, the state of the system changes as

$$
\boldsymbol{q} \rightarrow \boldsymbol{q}+q_{e} \boldsymbol{\Delta}_{ \pm \rho}, \quad\left(\boldsymbol{\Delta}_{\rho}\right)_{k}=-\delta_{k, n}+\delta_{k, m},
$$

where $q_{e}$ is the value of the elementary charge involved in all the possible transitions and $\boldsymbol{\Delta}_{-\rho}=-\boldsymbol{\Delta}_{\rho}$. The vector $\boldsymbol{\Delta}_{\rho}$ encodes to which conductors the device $\rho$ is connected and what the change in their number of charges is during the transitions. If a device $\rho$ is connected between one of the free conductors, $n$, and one with fixed voltage, the corresponding vector $\boldsymbol{\Delta}_{\rho}$ is given by

$$
\left(\boldsymbol{\Delta}_{\rho}\right)_{k}=\delta_{k, n}
$$

where the forward direction is chosen as the one leaving the conductor with fixed potential.

One can imagine more complex devices that involve three or more conductors in an irreducible way, for example, by taking one charge from conductor $m$ and one from $n$ and transporting them to conductor $o$. This kind of device can also be treated with the formalism we develop here, although most discussions are focused on two-terminal devices (that, however, might be controlled externally).

\section{A. Reduced incidence matrix, cycles, and conservation laws}

The vectors $\boldsymbol{\Delta}_{\rho>0}$ can be grouped in an $N \times M$ reduced incidence matrix:

$$
\boldsymbol{\Delta}=\left[\begin{array}{ccc}
\mid & \cdots & \mid \\
\boldsymbol{\Delta}_{1} & \cdots & \boldsymbol{\Delta}_{M} \\
\mid & \cdots & \mid
\end{array}\right]
$$

This matrix is analogous to the stoichiometric matrix in chemical reaction networks $[33,82,83]$. For closed circuits, it coincides with the full incidence matrix of the directed graph obtained by mapping conductors to nodes and twoterminal devices as directed edges (with the direction given by the forward one). The reduced incidence matrix for an open circuit is obtained from the one of the closed circuit by eliminating the rows corresponding to regulated conductors.

The right null eigenvectors of $\boldsymbol{\Delta}$ define cycles, i.e., sequences of transitions that leave the circuit state invariant:

$$
\Delta c_{\alpha}=0
$$

The elements of the vectors $\boldsymbol{c}_{\alpha}$ can always be chosen to be 0,1 , or -1 . The number of independent cycles is $N_{c}=\operatorname{dim}[\operatorname{Ker}(\boldsymbol{\Delta})]$. The left null eigenvectors of $\boldsymbol{\Delta}$ correspond to conservation laws, since if

$$
\boldsymbol{\ell}_{\nu}^{T} \boldsymbol{\Delta}=0,
$$

then the quantities

$$
L_{\nu}(\boldsymbol{q})=\boldsymbol{\ell}_{\nu}^{T} \boldsymbol{q}
$$

will not change under any transition; i.e., they are determined by the initial state of the circuit. The elements of $\ell_{\nu}$ can always be considered to be 0 or 1 . For each connected component of the full circuit in which no conductor is regulated, we have a conserved quantity that is just the total charge of the conductors in that component. In fact, these are the only conserved quantities. Thus, the number of independent conservation laws, $N_{l}=\operatorname{dim}\left[\operatorname{Ker}\left(\boldsymbol{\Delta}^{T}\right)\right]$, equals the number of closed connected components of the circuit.

Whenever a closed circuit is opened by connecting one of its conductors to a voltage source (see Fig. 1), we might either break a conservation law or create a new cycle. This possibility can be seen in the following way. The ranknullity theorem applied to the matrix $\boldsymbol{\Delta}$ can be expressed as

$$
N-N_{l}+N_{c}=M .
$$

This expression is valid for closed as well as for open circuits. Let us assume, however, that in the previous equation $N, N_{l}$, and $N_{c}$ correspond to the matrix $\boldsymbol{\Delta}$ of the circuit in which all the voltage sources are disconnected. Then, we connect $N_{r}$ voltage sources, and, thus, the number of conductors involved in the new matrix $\boldsymbol{\Delta}^{\prime}$ is now $N^{\prime}=N-N_{r}$. Applying the rank-nullity theorem to $\boldsymbol{\Delta}^{\prime}$, we obtain

$$
N^{\prime}-N_{l}^{\prime}+N_{c}^{\prime}=M .
$$

Subtracting the previous two equations, we see that

$$
N_{r}=N_{l}-N_{l}^{\prime}+N_{c}^{\prime}-N_{c} .
$$

Thus, the number of voltage sources connected to the circuit equals the number of broken conservation laws, $N_{l}-N_{l}^{\prime}$, plus the number of emergent cycles, $N_{c}^{\prime}-N_{c}$. This result is easily understood: If a previously closed component of the circuit is connected to a voltage source, its total charge ceases to be a conserved quantity. However, if we further connect another voltage source to another conductor of the same component, then a new cycle is created (the one in which a charge is injected by one source, transported through the component, and removed by the second source).

\section{B. Stochastic and deterministic dynamics}

At any given time, the state of the circuit is described by a probability distribution $P(\boldsymbol{q}, t)$ over the state space. It evolves according to the master equation 


$$
d_{t} P(\boldsymbol{q}, t)=\sum_{\rho}\left\{J_{-\rho}\left(\boldsymbol{q}+q_{e} \boldsymbol{\Delta}_{\rho}, t\right)-J_{\rho}(\boldsymbol{q}, t)\right\},
$$

where the probability currents are defined as

$$
J_{\rho}(\boldsymbol{q}, t)=\lambda_{\rho}(\boldsymbol{q}, t) P(\boldsymbol{q}, t) .
$$

They are simply the probability per unit time to observe a transition $\rho$ in state $\boldsymbol{q}$. The summation in Eq. (12) is over positive and negative values of $\rho$; i.e., it is over transitions and not over devices. The set of currents $J_{\rho}(\boldsymbol{q}, t)_{\rho= \pm 1, \ldots, \pm M}$ can be considered the components of a vector function of the state that we denote $\boldsymbol{J}(\boldsymbol{q}, t)$. We define the following operator over those functions:

$$
D_{q}^{\rho}[\boldsymbol{F}]=F_{-\rho}\left(\boldsymbol{q}+q_{e} \boldsymbol{\Delta}_{\rho}\right)-F_{\rho}(\boldsymbol{q}) .
$$

Then, $D_{q}^{\rho}[\boldsymbol{J}]$ is the net probability current arriving at state $\boldsymbol{q}$ corresponding to transitions $\pm \rho$, and the master equation reads

$$
d_{t} P(\boldsymbol{q}, t)=\sum_{\rho} D_{q}^{\rho}[\boldsymbol{J}]
$$

Note that the change in a scalar quantity $F(\boldsymbol{q})$ in a transition $\boldsymbol{q} \rightarrow \boldsymbol{q}+q_{e} \boldsymbol{\Delta}_{\rho}$ can also be expressed by trivially extending the operator $D_{q}^{\rho}[\cdot]$ to these functions:

$$
D_{\boldsymbol{q}}^{\rho}[F]=F\left(\boldsymbol{q}+q_{e} \boldsymbol{\Delta}_{\rho}\right)-F(\boldsymbol{q}) .
$$

The dynamical description based on the master equation in Eq. (12) is valid for timescales which are large compared to the time taken by each transition or conduction event, that here are considered to be instantaneous. This dynamics can be compared to the deterministic dynamics obtained by usual methods in circuit theory [84]. In those deterministic descriptions, the charges $q_{n}$ are considered to be continuous variables, and the charge vector $\boldsymbol{q}$ evolves according to the following differential equation:

$$
d_{t} \boldsymbol{q}=\sum_{\rho>0} \boldsymbol{\Delta}_{\rho} I_{\rho},
$$

where $I_{\rho}$ is the electric current associated to device $\rho$. The previous equation is closed by providing the $I-V$ curve characterization of all the devices and by Eq. (1) relating voltages and charges. For example, the current $I_{\rho}$ through a two-terminal device connected from conductor $n$ to conductor $m$ is considered to be a function $I_{\rho}\left(\Delta V_{n, m}\right)$ of the voltage drop $\Delta V_{n, m}=V_{n}-V_{m}$ (see Sec. III A). Then, $\Delta V_{n, m}$ can be expressed in terms of $\boldsymbol{q}$ by inverting Eq. (1). The relation between the deterministic and stochastic descriptions is nontrivial and is examined in the particular example of the CMOS inverter in Sec. VII A.

\section{Equilibrium states and detailed balance}

An equilibrium state $P_{\text {eq }}(q, t)$ of the circuit is defined as one in which the global detailed balance condition holds:

$$
D_{q}^{\rho}[\boldsymbol{J}]=0 \quad \forall \rho .
$$

By Eq. (15), if an equilibrium state exists, it is also a stationary one. In general, no equilibrium state exists. However, for closed and isothermal circuits, consistency with equilibrium thermodynamics demands the following Gibbs state to be an equilibrium one:

$$
P_{\text {Gibbs }}(\boldsymbol{q}, t)=Z^{-1} e^{-\beta E(\boldsymbol{q})} \prod_{\nu} \delta\left[L_{\nu}(\boldsymbol{q}), L_{\nu}\left(\boldsymbol{q}^{(i)}\right)\right],
$$

where $\delta[a, b]=1$ if $a=b$ and 0 otherwise, $\boldsymbol{q}^{(i)}$ is the initial state of the circuit, and $\nu$ runs over a set of independent conservation laws. The partition function $Z$ is such that $P_{\text {Gibbs }}(\boldsymbol{q}, t)$ is normalized and, thus, depends on the inverse temperature $\beta$ and the quantities $\left\{L_{\nu}\left(\boldsymbol{q}^{(i)}\right)\right\}$. More general equilibrium states can be obtained by mixing Gibbs distributions like Eq. (19) according to a distribution $P\left(\boldsymbol{q}^{(i)}\right)$ on the initial state.

The demand that $P_{\text {Gibbs }}(\boldsymbol{q}, t)$ must be an equilibrium state when the circuit is closed and isothermal imposes minimal conditions on the transition rates $\lambda_{ \pm \rho}(\boldsymbol{q}, t)$. These are the LDB conditions, which for closed circuits and isothermal settings are

$\log \frac{\lambda_{\rho}(\boldsymbol{q}, t)}{\lambda_{-\rho}\left(\boldsymbol{q}+q_{e} \boldsymbol{\Delta}_{\rho}, t\right)}=-\beta\left[E\left(\boldsymbol{q}+q_{e} \boldsymbol{\Delta}_{\rho}\right)-E(\boldsymbol{q})\right]$,

for each $\rho$. They can also be written as

$$
D_{q}^{\rho}[\log \lambda]=\beta D_{q}^{\rho}[E],
$$

where $\lambda(\boldsymbol{q}, t)$ is a vector function of the state with components $\left\{\lambda_{\rho}(\boldsymbol{q}, t)\right\}_{\rho= \pm 1, \ldots, \pm N}$ and the $\log (\cdot)$ function is applied elementwise. Thus, the rates $\lambda_{ \pm \rho}(\boldsymbol{q}, t)$ characterizing a given two-terminal device $\rho$ must fulfill the constraints imposed by Eq. (20). We now generalize the LDB conditions to open circuits and nonisothermal settings.

\section{Energy difference and local detailed balance}

We consider an open circuit in which some conductors have the potential fixed by voltage sources. In the same way we did with the charges, the vector $\boldsymbol{V}_{\mathbf{0}}$ is split into vectors $\boldsymbol{V}$ and $\boldsymbol{V}_{r}$, containing the voltages of the free and regulated conductors, respectively. We can then express the relation of Eq. (1) between all the charges and voltages as

$$
\left[\begin{array}{c}
\boldsymbol{q} \\
\boldsymbol{q}_{r}
\end{array}\right]=\left[\begin{array}{cc}
\boldsymbol{C} & \boldsymbol{C}_{m} \\
\boldsymbol{C}_{m}^{T} & \boldsymbol{C}_{r}
\end{array}\right]\left[\begin{array}{c}
\boldsymbol{V} \\
\boldsymbol{V}_{r}
\end{array}\right],
$$


where $\boldsymbol{C}_{r}$ is the $N_{r} \times N_{r}$ capacitance matrix of the regulated conductors, $\boldsymbol{C}$ is the $N \times N$ capacitance matrix of the free conductors, and $\boldsymbol{C}_{m}$ is the $N \times N_{r}$ matrix with the mutual capacitances between conductors of the two groups. The previous equation can be rewritten as

$$
\left[\begin{array}{c}
\boldsymbol{V} \\
\boldsymbol{q}_{r}
\end{array}\right]=\left[\begin{array}{cc}
\boldsymbol{C}^{-1} & -\boldsymbol{C}^{-1} \boldsymbol{C}_{m} \\
\boldsymbol{C}_{m}^{T} \boldsymbol{C}^{-1} & \boldsymbol{C}_{r}-\boldsymbol{C}_{m}^{T} \boldsymbol{C}^{-1} \boldsymbol{C}_{m}
\end{array}\right]\left[\begin{array}{c}
\boldsymbol{q} \\
\boldsymbol{V}_{r}
\end{array}\right],
$$

from where it is clear that, given the potentials $\boldsymbol{V}_{r}$, the charges $\boldsymbol{q}$ are enough to determine the rest of the variables, as discussed before. The total electrostatic energy is then

$$
\begin{aligned}
E(\boldsymbol{q}) & =\frac{1}{2}\left[\boldsymbol{q}^{T}, \boldsymbol{V}_{r}^{T}\right]\left[\begin{array}{c}
\boldsymbol{V} \\
\boldsymbol{q}_{r}
\end{array}\right] \\
& =\frac{1}{2} \boldsymbol{q}^{T} \boldsymbol{C}^{-1} \boldsymbol{q}+\frac{1}{2} \boldsymbol{V}_{r}^{T}\left(\boldsymbol{C}_{r}-\boldsymbol{C}_{m}^{T} \boldsymbol{C}^{-1} \boldsymbol{C}_{m}\right) \boldsymbol{V}_{r} .
\end{aligned}
$$

We are interested in computing how the total energy of the system (conductors plus sources) changes in a transition $\boldsymbol{q} \rightarrow \boldsymbol{q}+q_{e} \boldsymbol{\Delta}_{\rho}$. From the previous equation, we see that the change in electrostatic energy is

$$
\begin{aligned}
D_{\boldsymbol{q}}^{\rho}[E] & =E\left(\boldsymbol{q}+q_{e} \boldsymbol{\Delta}_{\rho}\right)-E(\boldsymbol{q}) \\
& =q_{e}^{2} \boldsymbol{\Delta}_{\rho}^{T} \boldsymbol{C}^{-1} \boldsymbol{\Delta}_{\rho} / 2+q_{e} \boldsymbol{q}^{T} \boldsymbol{C}^{-1} \boldsymbol{\Delta}_{\rho},
\end{aligned}
$$

which is independent of the voltages $\boldsymbol{V}_{r}$. In addition to this change, we need to consider the change in the energy stored in the voltage sources. This change can be computed as (minus) the work performed by them, which equals the charge transported from ground to the conductor to which each source is connected times its voltage. There are two different contributions to this work. First, the transition $\rho$ might directly involve one regulated conductor. If a charge $q_{e}$ arrives to the conductor fixed to a potential $V_{r}$, it needs to be removed, and for this removal the source must perform an amount of work given by $w_{r}=-q_{e} V_{r}$. Second, even if the transition does not involve any regulated conductor, changes in the distribution of charge among the free conductors can induce a charging of the regulated conductors. From Eq. (23), we see that the induced charge is $\delta \boldsymbol{q}_{r}=q_{e} \boldsymbol{C}_{m}^{T} \boldsymbol{C}^{-1} \boldsymbol{\Delta}_{\rho}$. It follows that the total amount of work performed by the sources during transition $\rho$ is

$$
\delta W_{\rho}=-q_{e} \boldsymbol{V}_{r}^{T} \boldsymbol{\Delta}_{\rho}^{r}+q_{e} \boldsymbol{V}_{r}^{T} \boldsymbol{C}_{m}^{T} \boldsymbol{C}^{-1} \boldsymbol{\Delta}_{\rho},
$$

where $\Delta_{\rho}^{r}$ is a vector encoding the change in the number of charges in the regulated conductors in transition $\rho$ (if no regulated conductor is involved in transition $\rho$, then $\left.\Delta_{\rho}^{r}=0\right)$. Thus, the change in the energy of the system and sources can be written as

$$
\delta Q_{\rho}(\boldsymbol{q})=D_{q}^{\rho}[E]-\delta W_{\rho}=D_{q}^{\rho}[\Phi]+q_{e} \boldsymbol{V}_{r}^{T} \Delta_{\rho}^{r},
$$

where we define the potential

$$
\Phi(\boldsymbol{q})=E(\boldsymbol{q})-\boldsymbol{V}_{r}^{T} \boldsymbol{C}_{m}^{T} \boldsymbol{C}^{-1} \boldsymbol{q} .
$$

The first term in the right-hand side in Eq. (27) is conservative, since its contribution vanishes in any cyclic sequence of transitions in the state space $\{\boldsymbol{q}\}$. The second contribution is not conservative, since its contribution does not vanish in cyclic transformations: Its value depends on how the regulated conductors are involved in the cycle. Also, we note that the gradient of the potential $\Phi(\boldsymbol{q})$ gives the voltage of the free conductors:

$$
\boldsymbol{V}(\boldsymbol{q})=\nabla_{\boldsymbol{q}} \Phi(\boldsymbol{q}),
$$

as can be verified from Eq. (23).

The quantity $\delta Q_{\rho}(\boldsymbol{q})$ is the energy required to perform the transition $\boldsymbol{q} \rightarrow \boldsymbol{q}+q_{e} \boldsymbol{\Delta}_{\rho}$. By conservation of energy, it must be provided by the environment of the device $\rho$, which we assume to be at thermal equilibrium at temperature $T_{\rho}$ (this assumption implies that the two conductors to which $\rho$ is connected should also be at temperature $T_{\rho}$ ). Therefore, $\delta Q_{\rho}(\boldsymbol{q})$ is the heat associated to device $\rho$ during the transition and corresponds to an entropy change in its environment equal to $-\delta Q_{\rho}(\boldsymbol{q}) / T_{\rho}$. Thus, the LDB condition now reads

$$
\log \frac{\lambda_{\rho}(\boldsymbol{q}, t)}{\lambda_{-\rho}\left(\boldsymbol{q}+q_{e} \boldsymbol{\Delta}_{\rho}, t\right)}=-\beta_{\rho} \delta Q_{\rho}(\boldsymbol{q}),
$$

for each $\rho$, where $\beta_{\rho}=\left(k_{b} T_{\rho}\right)^{-1}$. Equivalently,

$$
D_{q}^{\rho}[\log \lambda]=\beta_{\rho}\left(D_{q}^{\rho}[\Phi]+q_{e} \boldsymbol{V}_{r}^{T} \Delta_{\rho}^{r}\right) .
$$

For closed and isothermal settings, this condition reduces to Eq. (20).

\section{E. Infinite vs finite state spaces}

Certain types of circuits, in particular, single-electron devices, are such that the number of charges in a given conductor can take only a few distinct values. They can, therefore, be described by truncating their infinite state space $\{\boldsymbol{q}\}$ to the finite set of states relevant for the dynamics. This truncation does not compromise thermodynamic consistency. Such approaches can also be used to model singleelectron traps, which can be useful to model random telegraphic and $1 / f$ noise, as we discuss in Sec. III B 3.

For many other circuits, such truncation to a small state space is not possible. Infinite state spaces are indeed a crucial ingredient of devices displaying a macroscopic limit. This situation is the case of CMOS circuits, for instance, where, as the typical size of the transistors is increased, the number of electrons in each conductor becomes very large. In this case, the stochastic dynamics 
gives rise to a deterministic nonlinear dynamics [described by Eq. (17)], which enables the emergence of complex phenomena such as bistabilities, oscillations, and chaos. Studying fluctuations can become very expensive numerically, and many techniques suitable for finite state space (e.g., spectral methods) are not applicable anymore. Also, common approximation techniques, such as the secondorder truncation of the Kramers-Moyal expansion of Eq. (12) leading to Fokker-Planck or Langevin equations, are known to produce incorrect results $[85,86]$. One must, therefore, resort to more elaborate methods such as path integrals and large deviation techniques [87-91]. Such methods have been used to study stochastic chemical reaction networks [92], where similar problems are encountered [86], and were recently applied in electronics [90,91].

\section{MODELS FOR DEVICES}

\section{A. I-V curve characterization}

Two-terminal devices are usually characterized by measuring how the average electric current through them depends on the applied voltage across their terminals, as in Fig. 2. Modeling a device $\rho$ as a BPP with rates $\lambda_{+}$and $\lambda_{-}$ and applying the LDB condition of Eq. (30) to this simple case, we obtain

$$
\log \frac{\lambda_{+}(V)}{\lambda_{-}(V)}=\beta q_{e} V
$$

The net amount of charge going through the device in the forward direction between time $t$ and $t+\boldsymbol{\Delta} t$ is

$$
q(\boldsymbol{\Delta} t)=q_{e}\left[N_{+}(\boldsymbol{\Delta} t)-N_{-}(\boldsymbol{\Delta} t)\right],
$$

where $N_{ \pm}(\Delta t)$ are independent Poisson processes with rates $\lambda_{ \pm}(V)$. The average current is then

$$
\begin{aligned}
\langle I\rangle=\langle q(\boldsymbol{\Delta} t) / \Delta t\rangle & =q_{e}\left[\lambda_{+}(V)-\lambda_{-}(V)\right] \\
& =q_{e} \lambda_{+}(V)\left(1-e^{-\beta q_{e} V}\right) .
\end{aligned}
$$

Thus, the BPP modeling assumption and the LDB condition allow one to determine the rates $\lambda_{ \pm}(V)$ from the measurement of the $I-V$ curve alone, via Eqs. (32) and (34). In turn, from these rates, we can compute any statistical moment of the electric current $I(\Delta t)=q(\Delta t) / \Delta t$. Therefore, the full statistics of the process is completely

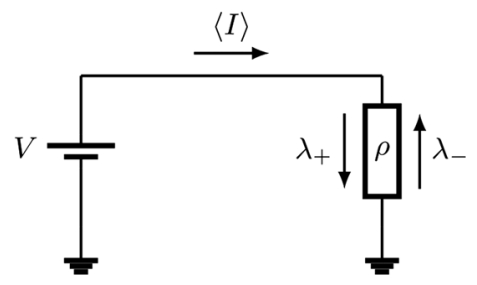

FIG. 2. $I-V$ characterization of a two-terminal device. determined by just the mean value $\langle I(\Delta t)\rangle$. In particular, the second central moment is $[20,21]$

$$
\begin{aligned}
\sigma_{I}^{2}(\boldsymbol{\Delta} t) & =\left\langle[I(\boldsymbol{\Delta} t)-\langle I(\boldsymbol{\Delta} t)\rangle]^{2}\right\rangle \\
& =\frac{q_{e}^{2}}{\boldsymbol{\Delta} t}\left[\lambda_{+}(V)+\lambda_{-}(V)\right] \\
& =\frac{q_{e}}{\boldsymbol{\Delta} t}\langle I(\Delta t)\rangle \operatorname{coth}\left(\beta q_{e} V / 2\right),
\end{aligned}
$$

which at variance with the first moment $\langle I(\Delta t)\rangle$ depends explicitly on the integration time $\Delta t$. This integration time is related via the Nyquist-Shannon sampling theorem to the frequency bandwidth $\Delta f=1 /(2 \Delta t)$ of the measurement. Thus, in the limit of large bias $\left(\beta q_{e} V \gg 1\right)$, we obtain the usual expression for shot noise:

$$
\sigma_{I}^{2}(\Delta t)=2 q_{e}\langle I\rangle \Delta f
$$

Then, in this context, shot noise appears as a direct consequence of the BPP assumption and of the LDB condition. For this reason, the fluctuations in circuits with elements that do not display shot noise cannot be faithfully described with this formalism. In the opposite limit where thermal effects dominate $\left(\beta q_{e} V \ll 1\right)$, we recover the usual expression for Johnson-Nyquist noise [55,93].

\section{B. Specific devices \\ 1. Tunnel junctions}

A tunnel junction is the simplest kind of device and the one for which the BPP model is more natural (in some regimes of operation) [40]. Here, we consider a tunnel junction consisting of a sufficiently small gap between two metallic islands at room temperature, such that electrons can tunnel through the gap. It typically displays an Ohmic $I-V$ curve $[40,94]:\langle I\rangle=V / R_{\mathrm{TJ}}$, where the tunnel junction resistance $R_{\mathrm{TJ}}$ can be computed from the specific properties of the metal conductors and the transmission coefficient of the junction. Using Eqs. (32) and (34), we obtain the rates

$$
\begin{aligned}
& \lambda_{+}(V)=\frac{V}{q_{e} R_{\mathrm{TJ}}} \frac{1}{1-e^{-\beta q_{e} V}}, \\
& \lambda_{-}(V)=\frac{V}{q_{e} R_{\mathrm{TJ}}} \frac{1}{e^{\beta q_{e} V}-1} .
\end{aligned}
$$

These expressions are well defined for any positive or negative value of the elementary charge $q_{e}$, and if it changes sign, then the roles $\lambda_{+}$and $\lambda_{-}$are just inverted. Many other different kinds of tunnel junctions exist, which can display strongly nonlinear $I-V$ curves depending on the spectral densities of the materials constituting the junction (see Ref. [40] for a quick review). 


\section{Diodes}

The characteristic curve of a $p$ - $n$ junction diode is often modeled via the ideal Shockley diode equation [95]:

$$
\langle I\rangle=I_{s}\left(e^{\beta q_{e} V}-1\right),
$$

where in this case $q_{e}$ is the positive electron charge and $I_{s}>0$ is the reversed bias saturation current. Then, the Poisson rates are given by

$$
\begin{aligned}
& \lambda_{-}(V)=I_{s} / q_{e}, \\
& \lambda_{+}(V)=\left(I_{s} / q_{e}\right) e^{V / V_{T}},
\end{aligned}
$$

where we define the thermal voltage

$$
V_{T}=\left(\beta q_{e}\right)^{-1}=k_{b} T / q_{e} .
$$

\section{MOS transistors in weak inversion}

MOS transistors are ubiquitous devices underlying most modern digital and analog electronics. An enhancementmode nMOS transistor like the one depicted in Fig. 3 has two typical modes of operation: (i) a saturation mode and (ii) a subthreshold or weak inversion mode (see Ref. [54] for a rigorous discussion of all the modes of operation). In the saturation mode, the transistor essentially behaves like a switch, allowing conduction between source $(S)$ and drain $(D)$ if the gate $(G)$ voltage is above a certain threshold $V_{\text {th }}$ [see Fig. 3(a)]. If $V_{G}<V_{\text {th }}$, then source-drain conduction is suppressed. However, whenever $V_{S} \neq V_{D}$, some small leakage current still flows, and its magnitude greatly depends on how far $V_{G}$ is below $V_{\text {th }}$. This mode is the subthreshold mode of operation, on which we focus in the following. To describe this mode, we consider the EnzKrummenacher-Vittoz model of the MOS transistor as developed in Ref. [53]. According to this model, the average drain current $\left\langle I_{D}\right\rangle$ can be naturally split into forward and reverse components given by

$$
\begin{aligned}
& \left\langle I_{D}^{f}\right\rangle=I_{0} e^{\left(V_{G}-V_{\text {th }}-n V_{S}\right) /\left(n V_{T}\right)}, \\
& \left\langle I_{D}^{r}\right\rangle=I_{0} e^{\left(V_{G}-V_{\text {th }}-n V_{D}\right) /\left(n V_{T}\right)},
\end{aligned}
$$

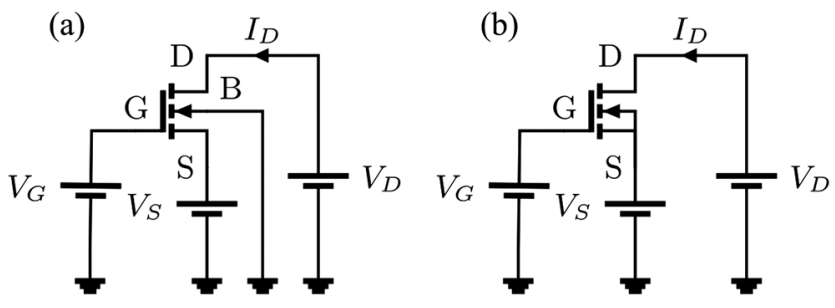

FIG. 3. An nMOS transistor. In (a), the bulk $(B)$ terminal is grounded, and all other voltages are measured with respect to it. This process allows one to preserve the symmetry between the source $(S)$ and drain $(D)$ terminals that is broken by connecting $S$ and $B$ together to obtain a three-terminal device like in (b). respectively, where the voltages and the current $I_{D}$ are defined as in Fig. 3(a). This model of the MOS transistor in subthreshold operation involves three parameters characterizing the device: the threshold voltage $V_{\text {th }}$, the "specific" current $I_{0}$, and the "slope factor" $n \geq 1$. All these parameters can be determined from a microscopic description of the device as explained in Ref. [53]. The total average drain current is then

$$
\begin{aligned}
\left\langle I_{D}\right\rangle & =\left\langle I_{D}^{f}\right\rangle-\left\langle I_{D}^{r}\right\rangle \\
& =I_{0} e^{\left(V_{G}-V_{\mathrm{th}}\right) /\left(n V_{T}\right)}\left(e^{-V_{S} / V_{T}}-e^{-V_{D} / V_{T}}\right) .
\end{aligned}
$$

In the previous expression, the symmetry of the device is preserved, since we see that the current $I_{D}$ is inverted if we interchange the roles of drain and source. For the more common three-terminal configuration of Fig. 3(b), the symmetry is broken, and the current is given by

$$
\left\langle I_{D}\right\rangle=I_{0} e^{\left(V_{G}-V_{S}-V_{\text {th }}\right) /\left(n V_{T}\right)}\left(1-e^{-\left(V_{D}-V_{S}\right) / V_{T}}\right) .
$$

The voltage bias driving this current is $V_{D}-V_{S}$, which plays the role of $V$ in the $I-V$ curve characterization. Using this last expression, we obtain the following Poisson rates:

$$
\begin{aligned}
& \lambda_{+}=\left(I_{0} / q_{e}\right) e^{\left(V_{G}-V_{S}-V_{\text {th }}\right) /\left(n V_{T}\right)}, \\
& \lambda_{-}=\left(I_{0} / q_{e}\right) e^{\left(V_{G}-V_{S}-V_{\text {th }}\right) /\left(n V_{T}\right)} e^{-\left(V_{D}-V_{S}\right) / V_{T}} .
\end{aligned}
$$

In principle, this model is accurate only for $\left\langle I_{D}^{f / r}\right\rangle \ll I_{0}$.

In a pMOS transistor, conduction between drain and source is increasingly allowed as the gate voltage becomes negative with respect to the body, contrarily to the nMOS transistor. However, all the expressions presented for the nMOS transistor are still valid for pMOS transistors provided that the signs of the currents and voltages are reversed, as Fig. 4 indicates.

In general treatments, the noise in MOS transistors is modeled by integrating infinitesimal Johnson-Nyquist sources along the drain-source channel $[53,54]$. However, for the subthreshold or weak-inversion mode in which we are interested, the results obtained in that way are fully compatible with those obtained from a simple BPP model as considered here [55]. This compatibility is not the case for other modes of operation. We note that this discussion concerns only the noise associated with the transport

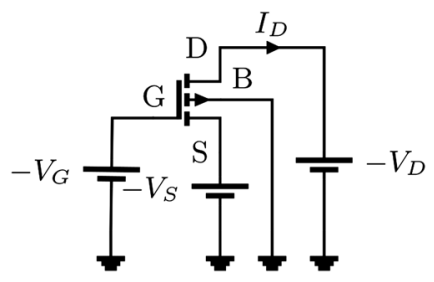

FIG. 4. Definition of voltage and current references for pMOS transistors. 
processes. Other kinds of noise associated with the presence of defects and charge traps $(1 / f$ and/or random telegraphic noise) play a role in MOS transistors [96-98] and are relevant at low frequency. Random telegraphic noise can nonetheless be modeled within the framework using individual charge traps, as briefly mentioned in Sec. II D. $1 / f$ can be modeled as well, as it can be generated by an ensemble of random telegraphic sources [99].

\section{CHARGING EFFECTS}

The previously discussed $I-V$ characterization of twoterminal devices allows one to determine the Poisson rates $\lambda_{ \pm \rho}$ for a given device $\rho$ in situations where the voltage across the device is kept fixed. In actual circuits, this voltage depends on the full state $\boldsymbol{q}$ of the circuit, in accordance with the relation of Eq. (1). Thus, the Poisson rates are functions $\lambda_{ \pm \rho}(\boldsymbol{q})$ of the full state. However, naive constructions of the functions $\lambda_{ \pm \rho}(\boldsymbol{q})$, based on the $I-V$ characterization and Eq. (1), fail to fulfill the LDB conditions. As a consequence, they lead to unphysical nonthermal stationary states for closed and isothermal circuits. To discuss and illustrate this situation, we revisit the well-known "Brillouin's paradox" [22]. Based on the analysis of this problem, we derive a procedure to construct the rates $\lambda_{ \pm \rho}(\boldsymbol{q})$ for arbitrary devices and circuits in such a way that the LDB conditions are always respected.

\section{A. Brillouin paradox}

We consider the circuit of Fig. 5: a closed and isothermal circuit consisting of a diode and a capacitor connected in parallel. At any given time, the voltage across the diode is equal to the capacitor voltage $V=q / C$, where $q$ is the total charge in the upper capacitor plate. Then, to construct the Poisson rates $\lambda_{ \pm}(q)$, we might consider the following procedure: To obtain the rate for a transition $q \rightarrow q \mp q_{e}$, we evaluate the fixed-voltage rates $\lambda_{ \pm}(V)$ of Eqs. (39) at the voltage preceding the transition (we refer to this assumption as a "naïve causality"). In this way, we have

$$
\begin{aligned}
& q \rightarrow q+q_{e}: \lambda_{-}^{\mathrm{nc}}(q)=I_{s} / q_{e}, \\
& q \rightarrow q-q_{e}: \lambda_{+}^{\mathrm{nc}}(q)=\left(I_{s} / q_{e}\right) e^{q /\left(C V_{T}\right)} .
\end{aligned}
$$

However, these rates do not fulfill the LDB condition of Eq. (20), that for this simple case reads

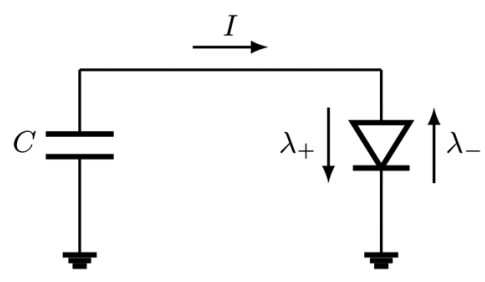

FIG. 5. Circuit illustrating the Brillouin paradox.

$$
\begin{aligned}
\log \frac{\lambda_{+}\left(q+q_{e}\right)}{\lambda_{-}(q)} & =-\beta\left[E(q)-E\left(q+q_{e}\right)\right] \\
& =\beta q_{e}\left[V+q_{e} /(2 C)\right] \\
& =\left(q+q_{e} / 2\right) /\left(C V_{T}\right)
\end{aligned}
$$

where $E(q)=q^{2} /(2 C)$ is the energy of the circuit. As a consequence, the stationary distribution corresponding to the transition rates $\lambda_{ \pm}^{c}(q)$ is

$$
P_{\mathrm{st}}^{c}(q) \propto e^{-(\beta / 2 C)\left(q^{2}+q_{e} q\right)},
$$

which deviates from the correct Gibbs equilibrium by a factor of $e^{-\beta q_{e} q /(2 C)}$. Since this factor is an uneven function of the charge, it follows that the stationary mean value of the charge in the capacitor is strictly below 0 . If this situation were the case, the capacitor could be disconnected from the diode and employed as a source of energy, and this process could, in principle, be repeated indefinitely. This apparent violation of the second law is essentially the Brillouin paradox and can be considered the electronic analog of a Brownian ratchet.

A way to solve this problem is to notice that the LDB condition of Eq. (46) would be fulfilled if the fixed-voltage rates $\lambda_{ \pm}(V)$ were evaluated not at the voltage before each transition but at the average of the voltage before and after the transition. Using this midpoint rule, we obtain the rates $[20,21]$

$$
\begin{aligned}
& q \rightarrow q+q_{e}: \lambda_{-}(q)=I_{s} / q_{e}, \\
& q \rightarrow q-q_{e}: \lambda_{+}(q)=\left(I_{s} / q_{e}\right) e^{\left(q-q_{e} / 2\right) /\left(C V_{T}\right)},
\end{aligned}
$$

which lead to the correct Gibbs equilibrium. Later, we show that the midpoint rule is valid, in general. This result means that it can be applied to the fixed-voltage rates $\lambda_{ \pm \rho}(V)$ of an arbitrary device $\rho$ to obtain thermodynamically consistent transition rates $\lambda_{ \pm \rho}(\boldsymbol{q})$ when this device is embedded in an arbitrary circuit. Although this rule seems to be at odds with the notion of causality, it is actually not: The probability of a transition naturally depends on the final state as well as on the initial one. For the naive notion of causality to be preserved, one should modify the characteristic $I-V$ curve of the device in question in a way that is context dependent. This modification, in turn, challenges the idea of modularity, i.e., the notion that the behavior of a device is not influenced by its environment and, therefore, can be plugged into different circuits without modifying its description, which is a basic assumption in the usual modeling of complex electronic circuits at the deterministic level. However, modifications to the characteristic curve of a device due to charging effects in its environment are well known in the study of single-electron devices, where the most explicit example is known as the Coulomb blockade 
effect $[39-43,94,100]$. In the following, we illustrate the charging effects in the context of the Brillouin paradox.

\section{B. Charging effects in the $I-V$ curve}

Let $\langle I\rangle_{V}$ be the average current for a given value of the capacitor voltage in the example in Fig. 5. According to the correct rates of Eqs. (48), it reads

$$
\langle I\rangle_{V}=I_{s}\left(e^{\left[V-q_{e} /(2 C)\right] / V_{T}}-1\right),
$$

which matches the characteristic $I-V$ function of Eq. (38) evaluated at a voltage shifted by $\delta V=q_{e} /(2 C)$. At the same time, the standard deviation of the voltage is $\sigma_{V} \simeq$ $\sqrt{k_{b} T / C}$. Then, charging effects are relevant whenever $\delta V$ is comparable to $V$ and $\sigma_{V}$. Consequently, in order to observe or employ these effects, as done, for example, in single-electron transistors, one needs to work either with nanoscopic circuits (in order to achieve low values of $C$ ) or at low temperatures (usually a combination of both). The characteristic curves of Eqs. (38) and (49) are compared in Fig. 6(a). We note that, counter to intuition, the mean value $\langle I\rangle_{V}$ does not vanish for $V=0$. This result seems to indicate that an initially empty capacitor would charge up when connected to the diode. However, in accordance with the second law, this anomaly is effectively neutralized by thermal fluctuations. This neutralization can be verified by computing the mean value of the current for the Gibbs equilibrium distribution $P_{\text {eq }}(q) \propto e^{-\beta q^{2} /(2 C)}$ :

$$
\langle I\rangle_{\mathrm{eq}}=\sum_{q} P_{\mathrm{eq}}(q)\langle I\rangle_{V=q / C}=0 .
$$

Also, for $0<V<\delta V$, we have $\langle I\rangle_{V}<0$, and, thus, it seems that for those values of $V$ the device is actually delivering power. However, this situation is not the case, since the actual voltage can take only the discrete values $V=n 2 \delta V$, with $n$ integer, as indicated with dots in Fig. 6(a). Finally, we note that if we take the limit $C \rightarrow \infty$ while fixing the voltage $V$, which corresponds to a model of a perfect voltage source, charging effects disappear (we go back to the picture in Fig. 2).

\section{General case}

Comparing Eqs. (32) and (30), we see that if $V$ in the fixed-voltage rates $\tilde{\lambda}_{ \pm}(V)$ is replaced by $\mp \delta Q_{ \pm \rho}(\boldsymbol{q}) / q_{e}$, then the resulting state-dependent rates automatically satisfy the LDB condition [recall the definition of $\delta Q_{ \pm \rho}(\boldsymbol{q})$ in Eq. (27) and note that it satisfies $\delta Q_{-\rho}\left(\boldsymbol{q}+q_{e} \boldsymbol{\Delta}_{\rho}\right)=$ $\left.-\delta Q_{\rho}(\boldsymbol{q})\right]$. Explicitly, we should consider (for $\rho>0$ )

$$
\lambda_{ \pm \rho}(\boldsymbol{q})=\tilde{\lambda}_{ \pm}\left[\mp \delta Q_{ \pm \rho}(\boldsymbol{q}) / q_{e}\right] .
$$

In turn, if device $\rho$ is connected to conductors $n$ and $m$ (with $n \rightarrow m$ as the forward direction), then we have
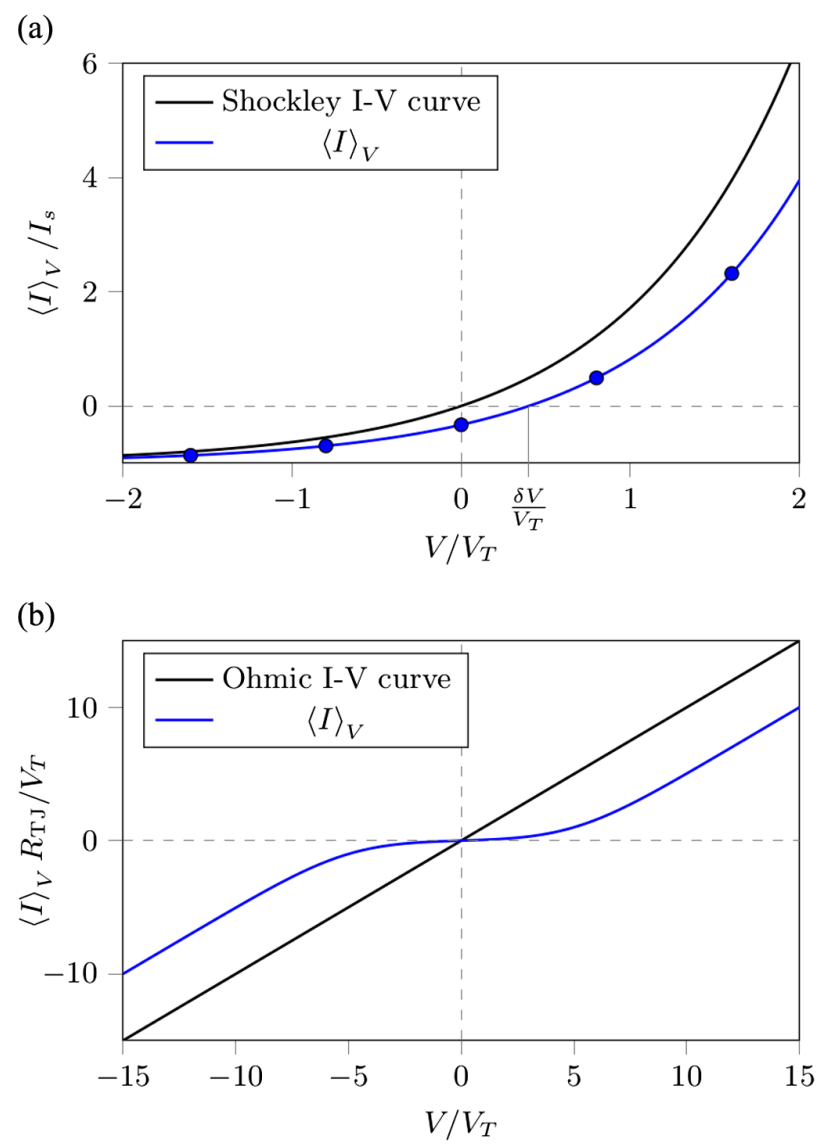

FIG. 6. Charging effects in the characteristic $I-V$ curve of a (a) Shockley diode $\left(\delta V / V_{T}=0.4\right)$ and (b) tunnel junction $\left(\delta V / V_{T}=5\right)$.

$$
\delta Q_{ \pm \rho}(\boldsymbol{q})=\mp q_{e} \overline{\boldsymbol{\Delta}}_{n m}^{ \pm \rho}(\boldsymbol{q}),
$$

where $\overline{\Delta V}_{n m}^{\rho}(\boldsymbol{q})$ is the average of the voltage difference $\Delta V_{n m}=V_{n}-V_{m}$ before and after the transition $\boldsymbol{q} \rightarrow \boldsymbol{q}+q_{e} \boldsymbol{\Delta}_{\rho}$. This result justifies the midpoint rule mentioned above and can be easily verified from the relation of Eq. (23) and the definition of $\Phi(\boldsymbol{q})$ in Eq. (28).

Particular care should be taken for the case of the MOS transistor (or, in general, with three-terminal devices that can be considered as externally controlled two-terminal devices). In this case, the fixed-voltage transition rates $\tilde{\lambda}_{ \pm}$ for the source-drain conduction do not depend only on the voltage difference $\Delta V_{D S}=V_{D}-V_{S}$ between those terminals, but also on the "control" voltage $\Delta V_{G S}=V_{G}-V_{S}$. Generalizing the rates of Eq. (44), we can write

$$
\begin{aligned}
& \tilde{\lambda}_{+}\left(\Delta V_{G S}, \Delta V_{D S}\right)=f\left(\Delta V_{G S}\right) g_{+}\left(\Delta V_{D S}\right), \\
& \tilde{\lambda}_{-}\left(\Delta V_{G S}, \Delta V_{D S}\right)=f\left(\Delta V_{G S}\right) g_{-}\left(\Delta V_{D S}\right),
\end{aligned}
$$

where the functions $f$ and $g_{ \pm}$are such that the following condition is satisfied: 


$$
\log \frac{\tilde{\lambda}_{+}\left(\Delta V_{G S}, \Delta V_{D S}\right)}{\tilde{\lambda}_{-}\left(\Delta V_{G S}, \Delta V_{D S}\right)}=\beta q_{e} \Delta V_{D S}
$$

To construct state-dependent rates satisfying the LDB condition of Eq. (30), we should not only replace $\Delta V_{D S}$ by its average before and after the transition but also do the same with the control parameter $\Delta V_{G S}$. Explicitly,

$$
\lambda_{ \pm \rho}(\boldsymbol{q})=\tilde{\lambda}_{ \pm}\left[\overline{\boldsymbol{\Delta}}_{G S}^{ \pm \rho}(\boldsymbol{q}), \overline{\boldsymbol{\Delta}}_{D S}^{ \pm \rho}(\boldsymbol{q})\right] .
$$

An analysis similar to the one for the diode in Sec. IV B also holds for more general circuits, including CMOS circuits. If $C$ is the typical capacitance at a given node of a circuit, the standard deviation of the voltage fluctuations at that node can be estimated by its equilibrium value $\sigma_{V}=\sqrt{k_{b} T / C}=\sqrt{V_{T} v_{e}}$, where we define the elementary voltage $v_{e}=q_{e} / C$ which represents the voltage change associated to a single charge jump. Values of $C$ as low as 50-100 aF can be attained in modern CMOS fabrication processes [101], that at room temperature lead to elementary voltages as high as $v_{e} \simeq 0.1 V_{T}$, and, thus, $\sigma_{V} \simeq 0.3 V_{T}$. Then, we see that in modern subthreshold or near-threshold applications it is not possible to neglect charging effects nor thermal fluctuations if the operating voltages are comparable to the thermal voltage $V_{T}$. This result, in turn, opens new possibilities that are explored in the example in Sec. VII B.

\section{Charging effects and nonlinearity}

Circuits containing devices with nonlinear $I-V$ curves are qualitatively different from circuits in which all devices are linear. For example, in linear $R L C$ networks (where the degrees of freedom are continuous and the stochastic dynamics is described by a Fokker-Planck equation), the dynamics of the mean or expected values of voltages and currents is decoupled from the dynamics of higher-order moments, and, therefore, it matches the deterministic dynamics [38]. This situation is not anymore the case if some nonlinear element is present. However, in the kind of discrete models we are considering here, charging effects can induce nonlinear behaviors even if the characteristic $I-V$ curves of all the devices in the circuit are linear, as is the case with tunnel junctions $[39,42,43]$. This situation is illustrated in Fig. 6(b), that is obtained in the same way as Fig. 6(a) for the diode. These induced nonlinearities are a resource that is exploited in the construction of singleelectron transistors and logic gates consisting of only small conductive islands and tunnel junctions between them [40]. However, in the macroscopic limit in which each conductor has many excess charges or for high temperatures, the nonlinear effects are washed out. Consequently, in those regimes, the expected values of voltages and currents in such circuits obey closed and linear equations of motion that match the ones obtained from the deterministic description of Eq. (17). The question about the relation between the stochastic and deterministic descriptions in general circuits is nontrivial and is not addressed here in full generality. It is analyzed for the particular example of the CMOS inverter in Sec. VII A. A general treatment will be considered elsewhere, based on large deviations theory $[90-92,102]$.

\section{STOCHASTIC THERMODYNAMICS}

So far, we have established the general stochastic description of electronic circuits, and we have shown how to construct the transition rates corresponding to different devices in a way that is thermodynamically consistent. In the following, we explore the general properties of this kind of models. We start by analyzing the energy balance and the entropy production; i.e., we establish the first and second laws. For this analysis, we first define the production of heat in each device and its relation to the electric current.

\section{A. Electrical currents and heat dissipation}

Let us consider the following pair of transitions, which are the inverse of each other:

$$
\boldsymbol{q} \underset{-\rho}{\stackrel{+\rho}{\rightleftharpoons}} \boldsymbol{q}+q_{e} \boldsymbol{\Delta}_{\rho}
$$

The average electric current corresponding to this pair of transitions is

$$
\left\langle I_{\rho}\right\rangle_{\boldsymbol{q}}=q_{e}\left[J_{\rho}(\boldsymbol{q}, t)-J_{-\rho}\left(\boldsymbol{q}+q_{e} \boldsymbol{\Delta}_{\rho}, t\right)\right]=-q_{e} D_{q}^{\rho}[\boldsymbol{J}] .
$$

Then, the net average electric current corresponding to device $\rho$ is

$$
\left\langle I_{\rho}\right\rangle=\sum_{q}\left\langle I_{\rho}\right\rangle_{q}=-q_{e} \sum_{q} D_{q}^{\rho}[\boldsymbol{J}] .
$$

In a similar way, the average rate at which heat is provided by the environment of device $\rho$ corresponding to that pair of transitions is

$$
\begin{aligned}
\left\langle\dot{Q}_{\rho}\right\rangle_{\boldsymbol{q}} & =\delta Q_{\rho}(\boldsymbol{q})\left[J_{\rho}(\boldsymbol{q})-J_{-\rho}\left(\boldsymbol{q}+q_{e} \boldsymbol{\Delta}_{\rho}, t\right)\right] \\
& =q_{e}^{-1} \delta Q_{\rho}(\boldsymbol{q})\left\langle I_{\rho}\right\rangle_{\boldsymbol{q}}
\end{aligned}
$$

where $\delta Q_{\rho}(\boldsymbol{q})$ is the change in energy of the system during transition $\rho$ and is given by Eq. (27). Note that, as is usual in stochastic thermodynamics but contrary to what is normally done in electronics, heat is defined as positive when it increases the energy of the system. Recalling Eq. (52), if device $\rho$ is connected to conductors $n$ and $m$, we can write

$$
\left\langle\dot{Q}_{\rho}\right\rangle_{\boldsymbol{q}}=-\overline{\boldsymbol{\Delta V}}_{n m}^{\rho}(\boldsymbol{q})\left\langle I_{\rho}\right\rangle_{\boldsymbol{q}}
$$


This expression is the stochastic version of the usual formula for Joule heating. Note that it is valid only at the level of transitions and that the average voltage difference is involved. The net heat rate associated to device $\rho$ is

$$
\begin{aligned}
\left\langle\dot{Q}_{\rho}\right\rangle & =\sum_{q}\left\langle\dot{Q}_{\rho}\right\rangle_{\boldsymbol{q}}=\sum_{\boldsymbol{q}} q_{e}^{-1} \delta Q_{\rho}(\boldsymbol{q})\left\langle I_{\rho}\right\rangle_{\boldsymbol{q}} \\
& =-\sum_{\boldsymbol{q}} D_{\boldsymbol{q}}^{\rho}[\Phi] D_{\boldsymbol{q}}^{\rho}[\boldsymbol{J}]+\left\langle I_{\rho}\right\rangle V_{r}^{T} \boldsymbol{\Delta}_{\rho}^{r}
\end{aligned}
$$

where we employ Eq. (27) to substitute $\delta Q_{\rho}(\boldsymbol{q})$.

\section{B. Balance of energy}

Let us consider the rate of change in the mean value of the potential $\Phi(\boldsymbol{q}, t)$ :

$$
d_{t}\langle\Phi\rangle=\sum_{\boldsymbol{q}} d_{t} P(\boldsymbol{q}, t) \Phi(\boldsymbol{q}, t)+\partial_{t}\langle\Phi\rangle .
$$

The explicit time dependence of $\Phi$ accounts for possible external controls of the parameters entering its definition, like the elements of the capacitance matrix or the voltages of the regulated conductors. Thus, the contribution $\partial_{t}\langle\Phi\rangle$ is interpreted as the rate of work done by this external control:

$$
\left\langle\dot{W}_{\Phi}\right\rangle=\partial_{t}\langle\Phi\rangle .
$$

Then, employing the master equation of Eq. (12), we can write

$$
\begin{aligned}
d_{t}\langle\Phi\rangle-\left\langle\dot{W}_{\Phi}\right\rangle & =\sum_{\rho, \boldsymbol{q}} D_{q}^{\rho}[\boldsymbol{J}] \Phi(\boldsymbol{q}, t) \\
& =-\frac{1}{2} \sum_{\rho, \boldsymbol{q}} D_{\boldsymbol{q}}^{\rho}[\boldsymbol{J}] D_{\boldsymbol{q}}^{\rho}[\Phi] \\
& =-\sum_{\rho>0} \sum_{\boldsymbol{q}} D_{\boldsymbol{q}}^{\rho}[\boldsymbol{J}] D_{q}^{\rho}[\Phi] \\
& =\sum_{\rho>0}\left\langle\dot{Q}_{\rho}\right\rangle-\sum_{\rho>0}\left\langle I_{\rho}\right\rangle \boldsymbol{V}_{r}^{T} \Delta_{\rho}^{r} .
\end{aligned}
$$

In the second and third lines of this equation, we use the symmetry $D_{q}^{\rho}[\cdot]=-D_{\boldsymbol{q}+q_{e} \boldsymbol{\Delta}_{\rho}}^{-\rho}[\cdot]$. Note that, at variance with the first line, the sums in the last line involve only positive values of $\rho$ and, therefore, can be considered as sums over devices. According to Sec. II D, the quantities

$$
\left\langle\dot{W}_{\rho}^{r}\right\rangle=-\left\langle I_{\rho}\right\rangle \boldsymbol{V}_{r}^{T} \boldsymbol{\Delta}_{\rho}^{r}
$$

are the average rates of work performed by the voltage sources corresponding to device $\rho$. In this way, we obtain the following energy balance for a general circuit:

$$
d_{t}\langle\Phi\rangle=\left\langle\dot{W}_{\Phi}\right\rangle+\sum_{\rho>0}\left\langle\dot{W}_{\rho}^{r}\right\rangle+\sum_{\rho>0}\left\langle\dot{Q}_{\rho}\right\rangle .
$$

\section{Entropy production}

To the probability distribution $P(\boldsymbol{q}, t)$, we assign the Shannon entropy:

$$
\langle S\rangle=-k_{b} \sum_{\boldsymbol{q}} P(\boldsymbol{q}, t) \log [P(\boldsymbol{q}, t)],
$$

which can be considered the average of the state-dependent entropy $S(\boldsymbol{q}, t)=-k_{b} \log [P(\boldsymbol{q}, t)]$. Its time derivative is

$$
\begin{aligned}
d_{t}\langle S\rangle & =-k_{b} \sum_{\rho} \sum_{q} D_{q}^{\rho}[\boldsymbol{J}] \log [P(\boldsymbol{q}, t)] \\
& =k_{b} \sum_{\rho>0} \sum_{q} D_{q}^{\rho}[\boldsymbol{J}] D_{q}^{\rho}[\log (P)] .
\end{aligned}
$$

As usual, this rate of entropy change can be split into two components:

$$
\begin{aligned}
d_{t}\langle S\rangle & =k_{b} \sum_{\rho>0} \sum_{q} D_{q}^{\rho}[\boldsymbol{J}] D_{q}^{\rho}[\log (P)] \\
& =k_{b} \sum_{\rho>0} \sum_{q}\left\{D_{q}^{\rho}[\boldsymbol{J}] D_{q}^{\rho}[\log (\boldsymbol{J})]-D_{q}^{\rho}[\boldsymbol{J}] D_{q}^{\rho}[\log (\lambda)]\right\} .
\end{aligned}
$$

Using the LDB condition in Eq. (31), the second term can be related to the average entropy production in the environment:

$$
\left\langle\dot{\Sigma}_{e}\right\rangle=k_{b} \sum_{\rho>0} \sum_{q} D_{q}^{\rho}[J] D_{q}^{\rho}[\log (\lambda)]=-k_{b} \sum_{\rho>0} \beta_{\rho}\left\langle\dot{Q}_{\rho}\right\rangle .
$$

Thus, combining the last two equations, we obtain the following expression for the total average irreversible entropy production:

$$
\langle\dot{\Sigma}\rangle \equiv d_{t}\langle S\rangle+\left\langle\dot{\Sigma}_{e}\right\rangle=k_{b} \sum_{\rho>0} \sum_{q} D_{q}^{\rho}[\boldsymbol{J}] D_{q}^{\rho}[\log (\boldsymbol{J})] \geq 0,
$$

which is explicitly positive. This expression constitutes a proof of the second law of thermodynamics in this context. We see that the entropy production $\langle\dot{\Sigma}\rangle$ vanishes if and only if $D_{q}^{\rho}[\boldsymbol{J}]=0$, i.e., if the state is an equilibrium one [see Eq. (18)]. The fact that $\langle\Sigma\rangle$ corresponds to the familiar concept of entropy production is further justified in the following.

\section{Isothermal conditions}

If the temperature of all the devices is the same, then we can split the total entropy production into a potential term and a work term. To see this split, we combine Eqs. (70) and (66) and write 


$$
T\left\langle\dot{\Sigma}_{e}\right\rangle=\left\langle\dot{W}_{\Phi}\right\rangle+\sum_{\rho>0}\left\langle\dot{W}_{\rho}^{r}\right\rangle-d_{t}\langle\Phi\rangle
$$

where $T$ is the common temperature of all devices. Then, we obtain

$$
\begin{aligned}
T\langle\dot{\Sigma}\rangle & =T d_{t}\langle S\rangle-d_{t}\langle\Phi\rangle+\left\langle\dot{W}_{\Phi}\right\rangle+\sum_{\rho>0}\left\langle\dot{W}_{\rho}^{r}\right\rangle \\
& =-d_{t}\langle F\rangle+\left\langle\dot{W}_{\Phi}\right\rangle+\sum_{\rho>0}\left\langle\dot{W}_{\rho}^{r}\right\rangle
\end{aligned}
$$

where we define the average free energy as

$$
\langle F\rangle=\langle\Phi\rangle-T\langle S\rangle
$$

Thus, the temperature times the total entropy production rate is expressed as a change in a thermodynamic potential plus the total work performed on the system. Integrating Eq. (73) and using the fact that $\langle\dot{\Sigma}\rangle>0$, we recover the usual statement of the second law: The amount of work that can be extracted from the system during an arbitrary transformation is limited by minus the free energy difference.

\section{Equilibrium states}

Because of the LDB conditions, for time-independent, closed, and isothermal systems, there is always an equilibrium state satisfying Eq. (18), and it is given by Eq. (19). However, equilibrium states could also exist under more general conditions. For example, if an isothermal and closed circuit is opened by connecting some or all connected components to a voltage source (in such a way that there is only one voltage source connected to each component), then there also exists an equilibrium state, in which no currents flow. To see this result, we notice that in such a case also the second term in Eq. (27) is conservative. In fact, we can write

$$
\begin{aligned}
\delta Q_{\rho}(\boldsymbol{q}) & =D_{q}^{\rho}[\Phi]+q_{e} \boldsymbol{V}_{r}^{T} \Delta_{\rho}^{r} \\
& =D_{q}^{\rho}\left[\Phi-\sum_{n_{p}} V_{n_{p}} L_{n_{p}}\right],
\end{aligned}
$$

where $n_{p}$ indexes the regulated conductors, $V_{n_{p}}$ is the voltage of conductor $n_{p}$, and

$$
L_{n_{p}}(\boldsymbol{q})=\boldsymbol{l}_{n_{p}}^{T} \boldsymbol{q}
$$

is the total charge of the free conductors in the connected component to which conductor $n_{p}$ belongs. Here, the vectors $l^{\prime}{ }_{n_{p}}$ can be constructed as the reduction to the space of free conductors of the left eigenvectors of the incidence matrix $\Delta$ of the full circuit, including the regulated conductors. We see then that in this case the energy change during a transition can be expressed as the change in a state function $\Psi(\boldsymbol{q})$ :

$$
\delta Q_{\rho}(\boldsymbol{q})=D_{q}^{\rho}[\Psi]
$$

with

$$
\Psi(\boldsymbol{q})=\Phi(\boldsymbol{q})-\sum_{n_{p}} V_{n_{p}} L_{n_{p}}(\boldsymbol{q}) .
$$

Thus, it follows that for isothermal conditions there exists an equilibrium state satisfying the global detailed balance conditions of Eq. (18). It is given by

$$
P_{\mathrm{eq}}(\boldsymbol{q})=Z^{-1} e^{-\beta \Psi(\boldsymbol{q})} \prod_{\nu_{c}} \delta\left[L_{\nu_{c}}(\boldsymbol{q}), L_{\nu_{c}}\left(\boldsymbol{q}^{(i)}\right)\right],
$$

where the index $\nu_{c}$ runs over the closed connected components of the circuit, $L_{\nu_{c}}(\boldsymbol{q})$ is the total charge of component $\nu_{c}$ as defined by Eq. (8), and $\boldsymbol{q}^{(i)}$ is the initial state. As with Eq. (19), the partition function $Z$ is such that $P_{\text {eq }}(\boldsymbol{q}, t)$ is normalized and, thus, depends on the conserved quantities $\left\{L_{\nu_{c}}\left(\boldsymbol{q}^{(i)}\right)\right\}$.

\section{E. Fundamental nonequilibrium forces}

Based on the previous discussion, we can now decompose the work performed by the sources into conservative and nonconservative contributions. For this decomposition, we first split the set of regulated conductors into two categories. For each of the open connected components in the full circuit, we arbitrarily select one of its regulated conductors. The conductors selected in this way are indexed by $n_{p}=1, \ldots, N_{p}$. As is clear later, the subindex $p$ stands for "potential." The total number $N_{p}$ of potential conductors can be easily seen to match the number of broken conservation laws as defined in Sec. II A. The rest of the regulated conductors are indexed by $n_{f}=1, \ldots, N_{f}$. In this case, the subindex $f$ stands for "force," and $N_{f}=$ $N-N_{p}$ equals the number of emergent cycles. In this way, to each transition $\rho$ involving a regulated conductor, we can assign (i) the voltage $V_{n_{r}(\rho)}$ of the regulated conductor involved in that transition and (ii) a reference voltage $V_{n_{p}(\rho)}$, that is the voltage of the regulated conductor $n_{p}$ that is selected as potential in the corresponding open connected component. Thus, the energy change during a transition $\rho$ involving a regulated conductor can be rewritten as

$$
\begin{aligned}
\delta Q_{\rho}(\boldsymbol{q})= & D_{q}^{\rho}[\Phi]+q_{e} \boldsymbol{V}_{r}^{T} \boldsymbol{\Delta}_{\rho}^{r} \\
= & D_{q}^{\rho}\left[\Phi-\sum_{n_{p}} V_{n_{p}} L_{n_{p}}\right] \\
& +q_{e}\left(\boldsymbol{\Delta}_{\rho}^{r}\right)_{n_{r}(\rho)}\left(V_{n_{r}(\rho)}-V_{n_{p}(\rho)}\right),
\end{aligned}
$$

where $L_{n_{p}}(\boldsymbol{q})$ is the total charge on the free conductors in the open connected component of conductor $n_{p}$, as defined in Eq. (76). Thus, the heat rate of device $\rho$ [Eq. (61)] can also be expressed as 


$$
\begin{aligned}
\left\langle\dot{Q}_{\rho}\right\rangle & =\sum_{\boldsymbol{q}} \frac{1}{q_{e}} \delta Q_{\rho}(\boldsymbol{q})\left\langle I_{\rho}\right\rangle_{\boldsymbol{q}} \\
& =-\sum_{\boldsymbol{q}} D_{q}^{\rho}[\Psi] D_{q}^{\rho}[\boldsymbol{J}]+\left\langle I_{\rho}\right\rangle\left(\boldsymbol{\Delta}_{\rho}^{r}\right)_{n_{r}(\rho)}\left(V_{n_{r}(\rho)}-V_{n_{p}(\rho)}\right),
\end{aligned}
$$

where $\Psi$ is the potential defined in Eq. (78). To each device $\rho$, we can assign a voltage difference $\Delta V_{\rho}=$ $-\left(\Delta_{\rho}^{r}\right)_{n_{r}(\rho)}\left(V_{n_{r}(\rho)}-V_{n_{p}(\rho)}\right)$. The minus sign in this definition is introduced in order to make $\Delta V_{\rho}$ positive whenever the reference voltage is the lowest one in each connected component, and the forward direction of device $\rho$ is the one leaving the regulated conductor. For an "internal" device not connected to any regulated conductor or for a device connected to a regulated conductor at the reference voltage, $\Delta V_{\rho}=0$. Then, we see that at most $N_{f}$ voltage differences $\Delta V_{\rho}$ can be different from zero. They are considered elements of a set $\left\{\boldsymbol{\Delta} V_{n_{f}}\right\}_{n_{f}=1, \ldots, N_{f}}$ of fundamental voltage differences or nonequilibrium forces. Therefore, the total heat rate can be written as

$$
\langle\dot{Q}\rangle=-\sum_{\rho>0} \sum_{q} D_{q}^{\rho}[\Psi] D_{q}^{\rho}[\boldsymbol{J}]-\sum_{n_{f}=1}^{N_{f}}\left\langle I_{n_{f}}\right\rangle \Delta V_{n_{f}},
$$

where $\Delta V_{n_{f}}$ is one of the $N_{f}$ fundamental nonequilibrium forces or voltage differences and $\left\langle I_{n_{f}}\right\rangle$ its associated electric current.

\section{F. Minimal decomposition of the isothermal entropy production}

For isothermal settings, the entropy production can be decomposed in a similar way as in Sec. V C 1, this time in terms of the grand potential

$$
\langle\Omega\rangle=\langle\Psi\rangle-T\langle S\rangle
$$

and the fundamental forces $\Delta V_{n_{f}}$. To see this similarity, we start by computing the change in the potential $\Psi$ :

$$
\begin{aligned}
d_{t}\langle\Psi\rangle-\partial_{t}\langle\Psi\rangle & =\sum_{q} d_{t} P(\boldsymbol{q}, t) \Psi(\boldsymbol{q}, t) \\
& =-\sum_{\rho>0} \sum_{q} D_{q}^{\rho}[\boldsymbol{J}] D_{q}^{\rho}[\Psi] .
\end{aligned}
$$

As before, we define a rate of work associated to the external control of the system:

$$
\left\langle\dot{W}_{\Psi}\right\rangle=\partial_{t}\langle\Psi\rangle \text {. }
$$

Note that this rate of work coincides with $\left\langle\dot{W}_{\Phi}\right\rangle$ in Eq. (63) only if the voltages of the regulated conductors are time independent. Now, combining the last two equations with Eq. (82) and recalling that for isothermal settings we have $T\left\langle\dot{\Sigma}_{e}\right\rangle=-\langle\dot{Q}\rangle$, we obtain

$$
T\left\langle\dot{\Sigma}_{e}\right\rangle=-d_{t}\langle\Psi\rangle+\left\langle\dot{W}_{\Psi}\right\rangle+\sum_{n_{f}}\left\langle I_{n_{f}}\right\rangle \Delta V_{n_{f}},
$$

that leads to the following expression for the irreversible entropy production:

$$
T\langle\dot{\Sigma}\rangle=-d_{t}\langle\Omega\rangle+\left\langle\dot{W}_{\Psi}\right\rangle+\sum_{n_{f}}\left\langle\dot{W}_{n_{f}}\right\rangle,
$$

where

$$
\left\langle\dot{W}_{n_{f}}\right\rangle=\left\langle I_{n_{f}}\right\rangle \Delta V_{n_{f}}
$$

is naturally defined as the work rate associated to the fundamental voltage difference $\Delta V_{n_{f}}$. We see that if the system is not driven $\left(\left\langle\dot{W}_{\Psi}\right\rangle=0\right)$ and there are no fundamental forces $\Delta V_{n_{f}}$, then $d_{t}\langle\Omega\rangle=-T \dot{\Sigma} \leq 0$. Also, from the fact that the capacitance matrix $C$ is positive definite, it follows that the thermodynamic potential $\langle\Omega\rangle$ is bounded from below. Thus, when $\left\langle\dot{W}_{\Psi}\right\rangle=\left\langle\dot{W}_{n_{f}}\right\rangle=0$, $\langle\Omega\rangle$ is a Lyapunov function that reaches a minimum at equilibrium.

\section{G. Nonequilibrium Landauer principle}

Let us consider a transformation between two arbitrary, possibly nonequilibrium, states $P^{(i)}(\boldsymbol{q})$ and $P^{(f)}(\boldsymbol{q})$. This transformation is driven by changing in time the parameters of the circuit (for example, the elements of the capacitance matrix or the properties of some of the devices) and/or by modifying the voltages of the regulated conductors. This transformation can induce a parametric driving of the potentials $\Phi$ and $\Psi$ (and, thus, also the free energies $F$ and $\Omega$ ), as well as a change in the nonequilibrium forces $\Delta V_{n_{f}}$ to which the system is subjected. In the following, we consider the time-dependent equilibrium state $P_{\text {eq }}(\boldsymbol{q}, t)$, which is just the equilibrium state of Eq. (79) corresponding to the parameters of the system at time $t$, and that will serve as a reference state. Also, given an arbitrary state $P(\boldsymbol{q}, t)$ [compatible with the conserved quantities $L_{\nu_{c}}\left(\boldsymbol{q}^{(i)}\right)$ ], we introduce its relative entropy with respect to the equilibrium state $P_{\text {eq }}(\boldsymbol{q}, t)$ :

$\mathcal{I}(t)=D\left(P \mid P_{\mathrm{eq}}\right)=\sum_{\boldsymbol{q}} P(\boldsymbol{q}, t) \log \left[P(\boldsymbol{q}, t) / P_{\mathrm{eq}}(\boldsymbol{q}, t)\right]$,

which in simple terms measures how much information should be provided in order to identify the state $P(\boldsymbol{q}, t)$ starting from $P_{\text {eq }}(\boldsymbol{q}, t)$. It vanishes if and only if $P(\boldsymbol{q}, t)=$ $P_{\text {eq }}(\boldsymbol{q}, t)$ and is always positive otherwise. By employing 
the explicit form of the equilibrium state $P_{\text {eq }}(\boldsymbol{q}, t)$, it is easy to see that the relative entropy can be computed as a difference between average free energies:

$$
k_{b} T \mathcal{I}(t)=\langle\Omega(t)\rangle-\langle\Omega(t)\rangle_{\mathrm{eq}},
$$

where $\langle\Omega(t)\rangle=\sum_{\boldsymbol{q}} P(\boldsymbol{q}, t) \Omega(\boldsymbol{q}, t) \quad$ is the nonequilibrium free energy and $\langle\Omega\rangle_{\mathrm{eq}}=\sum_{q} P_{\mathrm{eq}}(\boldsymbol{q}, t) \Omega(\boldsymbol{q}, t)=$ $-k_{b} T \log [Z(t)]$ is the equilibrium one. Using Eq. (90), we can rewrite Eq. (87) as

$$
\left\langle\dot{W}_{\Psi}\right\rangle+\sum_{n_{f}}\left\langle\dot{W}_{n_{f}}\right\rangle=k_{b} T d_{t} \mathcal{I}+d_{t}\langle\Omega\rangle_{\mathrm{eq}}+T\langle\dot{\Sigma}\rangle .
$$

Integrating this relation over time and using that $\langle\Sigma\rangle=$ $\int\langle\dot{\Sigma}\rangle d t \geq 0$, we obtain

$$
\left\langle W_{\Psi}\right\rangle+\sum_{n_{f}}\left\langle W_{n_{f}}\right\rangle \geq k_{b} T \Delta \mathcal{I}+\Delta\langle\Omega\rangle_{\mathrm{eq}} .
$$

Thus, the previous expression provides a bound for the amount of work necessary to perform (or that can be extracted during) a transformation between arbitrary states. Importantly, this bound explicitly takes into account the "information content" of the initial and final states with respect to the "uninformative" equilibrium, and it can be considered a general version of the Landauer principle [103]. The connection to the notion of logical or computational information is achieved by splitting the state space $\{\boldsymbol{q}\}$ into different logical subspaces. This splitting is done in Ref. [62], where a bound equivalent to Eq. (92) is derived.

The Landauer principle is commonly discussed in terms of physical memories that represent logical values as quasiequilibrium metastable states, of which the proper thermal equilibrium is a mixture [62,104-106]. However, it is important to notice that in some relevant kinds of electronic memories logical values are represented by nonequilibrium steady states (NESSs) that continuously produce entropy [for example, static random access memory (SRAM) cells or the probabilistic bit discussed in Sec. VII B]. Although the Landauer principle can anyway be applied to those cases, the bound obtained from the right-hand side of Eq. (92) does not take into account that continuous entropy production (or "housekeeping heat" [107]) or any other additional dissipation due to restrictions on the control parameters of the system [108]. Refinements of the Landauer principle based on lower bounds for the entropy production in nonadiabatic transformations can be obtained [67-72], but to the best of our knowledge the physics of computation with NESSs remains poorly explored.

\section{STOCHASTIC TRAJECTORIES AND FLUCTUATION THEOREMS}

In the previous sections, we study the average or expected values of relevant quantities like the energy, entropy, or work. In this section, we turn to a lower-level description based on single trajectories in the state space of the circuit, that allows us to formulate different fluctuation theorems. We closely follow the treatment in Ref. [33] for chemical reaction networks and of Ref. [28] for general Markov chains. Here, we present only the main results and the necessary definitions. Additional details about the derivations can be found in Supplemental Material [109].

We define a trajectory $\mathcal{Q}_{t}$ as a particular realization of the stochastic dynamics, from some initial time $\tau=0$ up to time $\tau=t$. Thus, a particular trajectory is fully characterized by its initial state $\boldsymbol{q}_{0}$, the set of transitions $\left\{\rho_{l}\right\}$ that take place up to time $t$, and the times $\left\{t_{l}\right\}$ at which they occur. The index $l$ takes the values $l=1, \ldots, N_{t}$, where $N_{t}$ is the number of transitions up to time $t$. All this information can be encoded in the trajectory probability current:

$$
j_{\rho}(\boldsymbol{q}, t)=\sum_{l=1}^{N_{t}} \delta\left[\rho, \rho_{l}\right] \delta\left[\boldsymbol{q}, \boldsymbol{q}_{t_{l}}\right] \delta\left(t-t_{l}\right),
$$

where $\boldsymbol{q}_{t}$ is the state immediately before instant $t$. Different trajectories occur with different probabilities. If the evolution of the system is well described by the master equation of Eq. (12), then the probability density $\mathcal{P}\left[\mathcal{Q}_{t}\right]$ of observing trajectory $\mathcal{Q}_{t}$ given that the initial state is $\boldsymbol{q}_{0}$ satisfies

$$
\mathcal{P}\left[\mathcal{Q}_{t}\right]=\prod_{l=0}^{N_{t}} e^{-\int_{t_{l}}^{t_{l+1}} \sum_{\rho} \lambda_{\rho}\left(\boldsymbol{q}_{\tau}, \tau\right) d \tau} \prod_{l=1}^{N_{t}} \lambda_{\rho_{l}}\left(\boldsymbol{q}_{t_{l}}, t_{l}\right),
$$

where we define $t_{N_{t}+1}=t$. The factors in the first product account for the probabilities of not having any transition during the periods $\left[t_{l}, t_{l+1}\right)$, while the factors in the second product are proportional to the probabilities of each of the jumps to take place. If we average Eq. (93) over all trajectories, then we recover the probability currents of Eq. (13).

The average quantities defined in the previous sections can be easily extended to individual trajectories. For example, the instantaneous electric current and power of device $\rho$ are, respectively,

$$
I_{\rho}(t)=-q_{e} \sum_{q} D_{q}^{\rho}[\boldsymbol{j}]
$$

and

$$
\begin{aligned}
\dot{Q}_{\rho}(t) & =-\sum_{q} \delta Q_{\rho}(\boldsymbol{q}) D_{\boldsymbol{q}}^{\rho}[\boldsymbol{j}] \\
& =-\sum_{q} D_{q}^{\rho}[\Psi] D_{q}^{\rho}[\boldsymbol{j}]-I_{\rho}(t) \Delta V_{n_{f}(\rho)}(t) .
\end{aligned}
$$


These are just the stochastic versions of the average quantities in Eqs. (81) and (57), respectively, and are obtained by simply replacing the average current vector $\boldsymbol{J}(\boldsymbol{q}, t)$ by the stochastic one $\boldsymbol{j}(\boldsymbol{q}, t)$, which is a vector function with components $\left\{j_{\rho}(\boldsymbol{q}, t)\right\}_{\rho= \pm 1, \ldots, \pm M} . \Delta V_{n_{f}}(t)$ is one of the fundamental nonequilibrium forces defined in Sec. VE.

The net change of a state function $f(\boldsymbol{q}, t)$ during a trajectory can be expressed in terms of the currents $j_{\rho}(\boldsymbol{q}, t)$ in the following way:

$$
\begin{aligned}
\boldsymbol{\Delta} f & =f\left(\boldsymbol{q}_{t}, t\right)-f\left(\boldsymbol{q}_{0}, 0\right) \\
& =\int_{0}^{t} d \tau\left\{\partial_{t} f\left(\boldsymbol{q}_{\tau}, \tau\right)+\sum_{\rho, \boldsymbol{q}} j_{\rho}(\boldsymbol{q}, \tau) D_{\boldsymbol{q}}^{\rho}\left[\left.f\right|_{\tau}\right]\right\} .
\end{aligned}
$$

Applying this expression to the potential $\Psi(\boldsymbol{q}, t)$ defined in Eq. (78), we can arrive at the following energy balance for a given trajectory:

$$
\Delta \Psi=W_{\Psi}+\sum_{n_{f}} W_{n_{f}}+\sum_{\rho>0} Q_{\rho}
$$

where

$$
W_{\Psi}=\int_{0}^{t} d \tau \partial_{t} \Psi\left(q_{\tau}, \tau\right)
$$

is the external driving work performed during the trajectory,

$$
Q_{\rho}=\int_{0}^{t} d \tau \dot{Q}_{\rho}(\tau)
$$

is the heat corresponding to device $\rho$, and

$$
W_{n_{f}}=\int_{0}^{t} d \tau I_{n_{f}}(\tau) \Delta V_{n_{f}}(\tau)
$$

is the work performed by the fundamental nonequilibrium force $\Delta V_{n_{f}}\left(I_{n_{f}}\right.$ is its associated electric current).

\section{A. Stochastic entropy and the integral fluctuation theorem}

As mentioned before, it is possible to define the entropy of a given state during a trajectory in the following way [110]:

$$
S(\boldsymbol{q}, t)=-k_{b} \log [P(\boldsymbol{q}, t)],
$$

where $P(\boldsymbol{q}, t)$ is the solution of the master equation in Eq. (12) for a given initial distribution $P(\boldsymbol{q}, 0)$. The entropy flow, i.e., the production of entropy in the environment during a given trajectory, is

$$
\begin{aligned}
\Sigma_{e}\left(\mathcal{Q}_{t}\right) & =-k_{b} \sum_{\rho} \beta_{\rho} Q_{\rho}(t) \\
& =-k_{b} \int_{0}^{t} d \tau \sum_{\rho, \boldsymbol{q}} j_{\rho}(\boldsymbol{q}, \tau) D_{\boldsymbol{q}}^{\rho}\left[\left.\log (\lambda)\right|_{\tau}\right] .
\end{aligned}
$$

Then, the total entropy production during the trajectory is

$$
\begin{aligned}
\Sigma\left(\mathcal{Q}_{t}\right)= & -k_{b} \log \left(\frac{P\left(\boldsymbol{q}_{t}, t\right)}{P\left(\boldsymbol{q}_{0}, 0\right)}\right) \\
& -k_{b} \int_{0}^{t} d \tau \sum_{\rho, \boldsymbol{q}} j_{\rho}(\boldsymbol{q}, \tau) D_{\boldsymbol{q}}^{\rho}\left[\left.\log (\lambda)\right|_{\tau}\right] .
\end{aligned}
$$

It can be verified that the time derivatives of the averages $\left\langle\Sigma_{e}\right\rangle$ and $\langle\Sigma\rangle$ over all trajectories match the entropy production rates $\left\langle\dot{\Sigma}_{e}\right\rangle$ and $\langle\dot{\Sigma}\rangle$ defined in Eqs. (70) and (71). Unlike its average $\langle\Sigma\rangle$, the entropy production $\Sigma$ of a given trajectory is not always positive. However, the fluctuations of $\Sigma$ are bound to satisfy a general integral fluctuation theorem. This fundamental result is expressed as the following equality:

$$
\left\langle e^{-\Sigma / k_{b}}\right\rangle=1
$$

where the average is taken over all trajectories $\mathcal{Q}_{t}$. In simple terms, this equality states that positive values of the full entropy production are more probable than negative ones. Accordingly, from this result and Jensen's inequality the usual statement of the second law follows: $\langle\Sigma\rangle \geq 0$. Equation (105) is valid for transient or steady state dynamics, in autonomous or time-dependent circuits.

\section{B. Detailed fluctuation theorems}

Equation (105) is only one of several fluctuation theorems. Under certain conditions, other quantities different from the full entropy production satisfy more stringent constraints. For example, in isothermal settings where $\beta_{\rho}=\left(k_{b} T\right)^{-1}$ for all $\rho$, it is possible to obtain the following detailed fluctuation theorem (DFT):

$$
\frac{P\left(\left\{W_{n_{f}}\right\}, W_{\Psi}\right)}{P^{\dagger}\left(\left\{-W_{n_{f}}\right\},-W_{\Psi}\right)}=\exp \left[\left(W_{\Psi}+\sum_{n_{f}} W_{n_{f}}\right) /\left(k_{b} T\right)\right] .
$$

In this expression, $P\left(\left\{W_{n_{f}}\right\}, W_{\Psi}\right)$ is the probability to observe the values $\left\{W_{n_{f}}\right\}$ of work for each of the fundamental forces and of $W_{\Psi}$ for the driving work during a forward protocol. This protocol consists of the initialization of the system state at $t=0$ according to the equilibrium state of Eq. (79) and its subsequent evolution according to the transition rates $\lambda(\boldsymbol{q}, \tau)$ up to time $t$ (the explicit dependence of the rates on $\tau$ takes into account a possible external manipulation of the circuit parameters, leading to an 
inhomogeneous time evolution). Analogously, the quantity $P^{\dagger}\left(\left\{-W_{n_{f}}\right\},-W_{\Psi}\right)$ is the probability to observe the work values $\left\{-W_{n_{f}}\right\}$ and $-W_{\Psi}$ during the corresponding backward protocol, in which the system is initialized in a state drawn from the equilibrium distribution of Eq. (79) (this time corresponding to the circuit parameters at time $t$ ) and evolves according to the rates $\lambda(\boldsymbol{q}, t-\tau)$.

The previous result holds for arbitrary times $t$, under the condition that the initial state is an equilibrium one. A complementary relation can be obtained, which is valid only asymptotically (that is, for long times) but irrespective of the initial distribution (under the additional assumption that the circuit state is bounded to a finite region of the state space). It reads

$$
\frac{P\left(\left\{\overline{\dot{W}}_{n_{f}}\right\}, \overline{\dot{W}}_{\Psi}\right)}{P^{\dagger}\left(\left\{-\overline{\dot{W}}_{n_{f}}\right\},-\overline{\dot{W}}_{\Psi}\right)} \simeq \exp \left[t\left(\overline{\dot{W}}_{\Psi}+\sum_{n_{f}} \overline{\dot{W}}_{n_{f}}\right) /\left(k_{b} T\right)\right],
$$

where we define the average work rates $\overline{\dot{W}}_{\Psi}=t^{-1} W_{\Psi}$ and $\overline{\dot{W}}_{n_{f}}=t^{-1} W_{n_{f}}$. Note that in the case in which the voltage differences $\Delta V_{n_{f}}$ are constant we have $W_{n_{f}}=\bar{I}_{n_{f}} \Delta V_{n_{f}}$, where $\bar{I}_{n_{f}}=t^{-1} \int_{0}^{t} d \tau I_{n_{f}}(\tau)$ are the average associated currents during the trajectory, and, therefore, Eq. (107) can be easily expressed in terms of the probabilities $P\left(\left\{\bar{I}_{n_{f}}\right\}, \bar{W}_{\Psi}\right)$. If there is no external driving of the circuit parameters, then $P(\cdot)=P^{\dagger}(\cdot)$.

The fluctuation theorems in Eqs. (106) and (107) express fundamental symmetries of energy exchange processes. For example, the novel thermodynamic uncertainty relations [111] or the usual Onsager relations (as well as their nonlinear extension [57]) can be recovered from them. For completeness, a general proof of these and other fluctuation theorems is given in Supplemental Material [109].

\section{APPLICATIONS}

\section{A. The CMOS inverter}

The inverter or NOT gate is the most elementary logic gate. It has a single logical input, which is negated in its only output. A diagram of a possible implementation of this gate with MOS transistors is shown in Fig. 7(a). It is composed by one pMOS (top) and one nMOS (bottom) transistor, with common drain and gate terminals. The device is powered by applying a voltage difference $V_{\mathrm{dd}}-V_{\mathrm{ss}}$ between source terminals. When the voltage in the input is $V_{\text {in }}<\left(V_{\mathrm{dd}}+V_{\mathrm{ss}}\right) / 2$, conduction in the nMOS transistor is suppressed, while it is enhanced in the pMOS transistor, and, therefore, the output voltage $V_{\text {out }}$ rapidly approaches $V_{\mathrm{dd}}$. The situation is reversed for $V_{\text {in }}>\left(V_{\text {dd }}+V_{\text {ss }}\right) / 2$, as shown in Fig. 7(b).

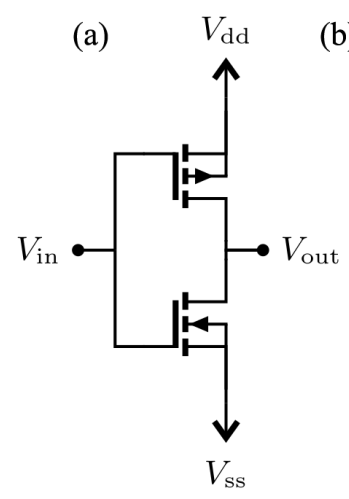

(b)

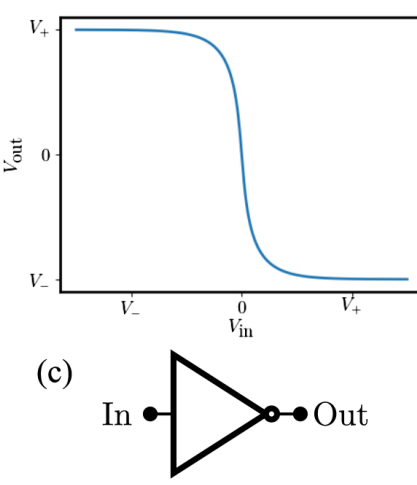

FIG. 7. (a) Common implementation of a NOT gate with CMOS technology. (b) Typical deterministic output voltage as a function of the input (for $V_{\mathrm{ss}}=-V_{\mathrm{dd}}$ ). (c) Logical symbol for the NOT gate.

Now, we explain how to build a stochastic model of the inverter within our formalism. The first step is to model the MOS transistor as an externally controlled conduction channel, with associated capacitances. For example, the nMOS transistor at the bottom of the diagram in Fig. 7(a) can be represented as in Fig. 8. There, the transistor is represented as an externally controlled conduction channel between source and drain. The Poisson rates $\lambda_{ \pm}^{n}$ associated to that channel are constructed as explained in Sec. IV C and depend on the gate to source voltage as well as on the drain to source voltage. The gate-body interface is modeled as a capacitor of capacitance $C_{g}$, and another capacitor $C_{o}$ takes into account the output capacitance of the transistor. Using this mapping, we can model the full inverter with the diagram in Fig. 9. In turn, this diagram corresponds to a set of four conductors, in which three of them are regulated by voltage sources, as shown in Fig. 10. The relation between the charges and voltages in this system is given by
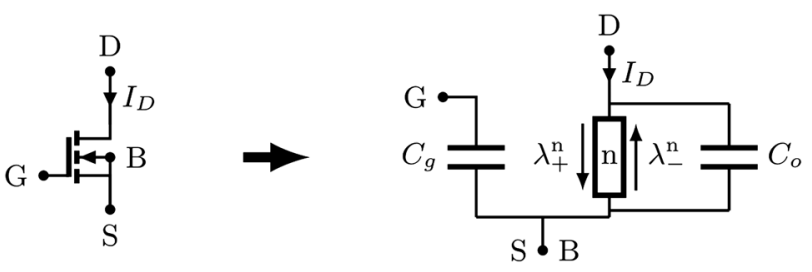

FIG. 8. Model of an nMOS transistor as an externally controlled conduction channel between source and drain, with associated Poisson rates $\lambda_{ \pm}^{n}$. The gate-body interface is represented as a capacitor $C_{g}$, and another capacitor $C_{o}$ takes into account the output capacitance. This model is just a minimal model and does not pretend to be realistic. Other parasitic capacitances could also be taken into account, for example, between drain and gate, but a proper description of them must take into account the physical dimensions of the device. 


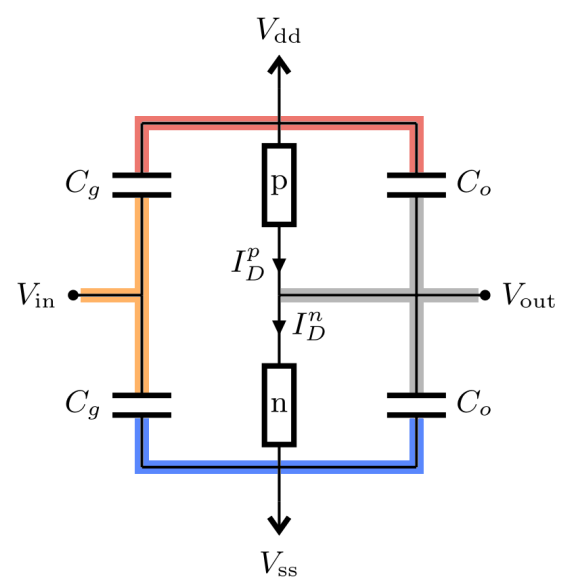

FIG. 9. A possible model of the CMOS inverter. The different conductors in the circuit (regions with the same potential) are identified with different colors (see Fig. 10).

$\left[\begin{array}{c}q_{\text {out }} \\ q_{\text {in }} \\ q_{\mathrm{dd}} \\ q_{\mathrm{ss}}\end{array}\right]=\left[\begin{array}{c|ccc}2 C_{o} & 0 & -C_{o} & -C_{o} \\ \hline 0 & 2 C_{g} & -C_{g} & -C_{g} \\ -C_{o} & -C_{g} & C_{o}+C_{g} & 0 \\ -C_{o} & -C_{g} & 0 & C_{o}+C_{g}\end{array}\right]\left[\begin{array}{c}V_{\text {out }} \\ V_{\text {in }} \\ V_{\mathrm{dd}} \\ V_{\mathrm{ss}}\end{array}\right]$,

where $q_{\text {out }}$ is the charge of the only free conductor (the output of the gate) and to which we refer simply as $q$ in the following. By comparison with Eq. (22), we can extract the blocks $\boldsymbol{C}, \boldsymbol{C}_{m}$, and $\boldsymbol{C}_{r}$ of the capacitance matrix (separated by lines in the previous expression). Using that and Eq. (22), we obtain the internal energy of the circuit as a function of the only degree of freedom $q$ :

$E(q)=\frac{q^{2}}{4 C_{o}}+\frac{C_{o}}{4} \Delta V^{2}+\frac{C_{g}}{2}\left[\left(V_{\mathrm{in}}-V_{\mathrm{dd}}\right)^{2}+\left(V_{\mathrm{in}}-V_{\mathrm{ss}}\right)^{2}\right]$,

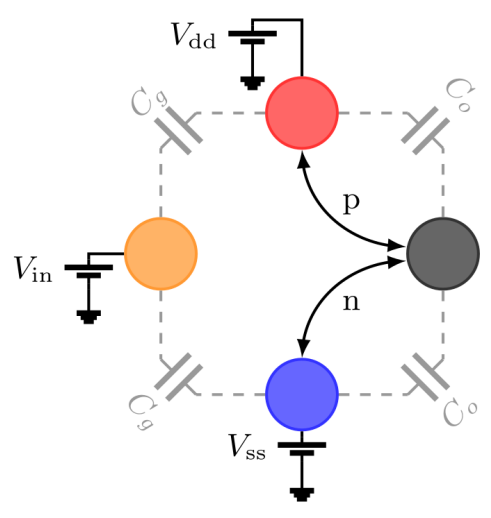

FIG. 10. Representation of the CMOS inverter as a set of three regulated and one free conductors and two conduction channels. The capacitors represent the mutual capacitances between them. with $\Delta V=V_{\mathrm{dd}}-V_{\mathrm{ss}}$. In the same way, from Eq. (28), we can obtain the potential

$$
\Phi(q)=E(q)+q\left(V_{\mathrm{dd}}+V_{\mathrm{ss}}\right) / 2,
$$

and by taking its gradient we obtain the output voltage as a function of $q$ :

$$
V_{\text {out }}(q)=q /\left(2 C_{o}\right)+\left(V_{\mathrm{dd}}+V_{\mathrm{ss}}\right) / 2 .
$$

Also, by selecting $V_{\mathrm{ss}}$ as the reference voltage to construct the potential $\Psi$ [Eq. (78)], we find

$$
\Psi(q)=\Phi(q)-q V_{\mathrm{ss}}=E(q)+q \Delta V / 2
$$

and that $\Delta V$ as defined above is the only nonequilibrium force (with $I_{p}$, the current through the pMOS transistor, as the associated current).

According to Eq. (98), the energy balance for this circuit at the trajectory level is

$$
d_{t} \Psi=\dot{Q}_{n}+\dot{Q}_{p}+I_{p} \Delta V
$$

where $\dot{Q}_{n}$ and $\dot{Q}_{p}$ are the heat currents associated to each of the transistors. The irreversible entropy production is given by Eq. (87) and for this case reads

$$
T\langle\dot{\Sigma}\rangle=-d_{t}\langle\Omega\rangle+\left\langle I_{p}\right\rangle \boldsymbol{\Delta} V
$$

where $T$ is the temperature of both transistors and $\langle\Omega\rangle=$ $\langle\Psi\rangle-T\langle S\rangle$ [we assume time-independent voltages, so the driving contribution of Eq. (85) is not present]. As can be seen from the two previous equations, for steady state conditions $\left(d_{t}\langle\Psi\rangle=d_{t}\langle S\rangle=0\right)$, the entropy production $\langle\dot{\Sigma}\rangle$ reduces to the entropy flow $\left\langle\dot{\Sigma}_{e}\right\rangle$, and we recover the usual expression:

$$
T\langle\dot{\Sigma}\rangle=-\left\langle\dot{Q}_{n}\right\rangle-\left\langle\dot{Q}_{p}\right\rangle=\left\langle I_{p}\right\rangle \Delta V
$$

We now build the transition rates associated to both transistors according to the procedure in Sec. IV C. We begin with the nMOS transistor. The voltage difference between drain and source is $\Delta V_{D S}=V_{\text {out }}-V_{\text {ss }}$, and, thus, its average during the transition $q \rightarrow q \pm q_{e}$, with rates $\lambda_{\mp}^{n}(q)$, is $\overline{V_{D S}}=\left(q \pm q_{e} / 2\right) /\left(2 C_{o}\right)+\Delta V / 2$. For the pMOS transistor, the voltage difference between source and drain is $\Delta V_{S D}=V_{\mathrm{dd}}-V_{\text {out }}$ (recall that for pMOS transistors the references for voltage and currents are reversed), and its average for the same transitions, this time with rates $\lambda_{ \pm}^{p}(q)$, is $\overline{V_{S D}}=-\left(q \pm q_{e} / 2\right) /\left(2 C_{o}\right)+\Delta V / 2$. Then, via the procedure in Sec. IV C and the fixed-voltage rates in Eq. (44), we obtain the transition rates 


$$
\begin{aligned}
\lambda_{+}^{n}(q) & =\left(I_{0} / q_{e}\right) e^{\left(V_{\text {in }}-V_{\mathrm{ss}}-V_{\mathrm{th}}\right) /\left(n V_{T}\right)}, \\
\lambda_{-}^{n}(q) & =\lambda_{+}^{n}(q) e^{-\left[\left(q+q_{e} / 2\right) /\left(2 C_{o}\right)+\Delta V / 2\right] / V_{T}}
\end{aligned}
$$

for the nMOS transistor and

$$
\begin{aligned}
& \lambda_{+}^{p}(q)=\left(I_{0} / q_{e}\right) e^{\left(V_{\mathrm{dd}}-V_{\mathrm{in}}-V_{\mathrm{th}}\right) /\left(n V_{T}\right)}, \\
& \lambda_{-}^{p}(q)=\lambda_{+}^{p}(q) e^{-\left[-\left(q-q_{e} / 2\right) /\left(2 C_{o}\right)+\Delta V / 2\right] / V_{T}}
\end{aligned}
$$

for the pMOS. Thus, the master equation for the distribution $P(q, t)$ reads

$$
\begin{aligned}
d_{t} P(q, t)= & P\left(q-q_{e}, t\right)\left[\lambda_{-}^{n}\left(q-q_{e}\right)+\lambda_{+}^{p}\left(q-q_{e}\right)\right] \\
& +P\left(q+q_{e}, t\right)\left[\lambda_{+}^{n}\left(q+q_{e}\right)+\lambda_{-}^{p}\left(q+q_{e}\right)\right] \\
& -P(q, t)\left[\lambda_{-}^{n}(q)+\lambda_{+}^{n}(q)+\lambda_{-}^{p}(q)+\lambda_{+}^{p}(q)\right] .
\end{aligned}
$$

The master equation can be employed, for example, to find the steady state for given voltages $V_{\mathrm{in}}, V_{\mathrm{dd}}$, and $V_{\mathrm{ss}}$. As shown in Supplemental Material [109] and the steady state is uniquely determined by the following recurrence relation:

$$
P_{\mathrm{st}}(q)=\frac{\alpha_{p}+\alpha_{n} \gamma e^{-\left(q-q_{e}\right) / q_{T}}}{\alpha_{n}+\alpha_{p} \gamma e^{q / q_{T}}} P_{\mathrm{st}}\left(q-q_{e}\right),
$$

where we define the constants

$$
\alpha_{n}=e^{\left(V_{\text {in }}-V_{\mathrm{ss}}\right) /\left(n V_{T}\right)}, \quad \alpha_{p}=e^{\left(V_{\mathrm{dd}}-V_{\text {in }}\right) /\left(n V_{T}\right)},
$$

and

$\gamma_{0}=e^{-\Delta V /\left(2 V_{T}\right)}, \quad q_{T}=2 C_{o} V_{T}, \quad \gamma=\gamma_{0} e^{-q_{e} /\left(2 q_{T}\right)}$.
From Eq. (119), it follows that the mean value $\langle q\rangle_{\text {st }}$ can be obtained from the positive root $x$ of

$$
\alpha_{p} \gamma_{0} e^{a+b} x^{2}+\left(\alpha_{n}-\alpha_{p}\right) x-\alpha_{n} \gamma_{0} e^{a-b}=0,
$$

as $\langle q\rangle_{\mathrm{st}}=q_{T} \log (x)$. The constants $a$ and $b$ are such that

$$
\left\langle e^{ \pm\left(q-\langle q\rangle_{\mathrm{st}}\right) / q_{T}}\right\rangle_{\mathrm{st}}=e^{q_{e} /\left(2 q_{T}\right)} e^{a \pm b},
$$

where the mean value is taken on the stationary state given by Eq. (119) (see Supplemental Material [109]). Thus, $a$ and $b$ quantify the fluctuations of the output charge around the mean value. They are defined so that if the stationary state is a thermal equilibrium state (as it is for $V_{\mathrm{dd}}=V_{\mathrm{ss}}$ ), then $a=b=0$. The constant $a$ is a measure of how the even moments of $P_{\mathrm{st}}(q)$ around the mean value deviate from those corresponding to equilibrium, while $b$ is the same for the odd moments. We see then that Eq. (122) determines how the nonequilibrium fluctuations, characterized by $a$ and $b$, affect the expected output of the gate $\langle q\rangle_{\text {st }}$. If we assume that the fluctuations are always compatible with thermal equilibrium (i.e., if $a=b=0$ ), then Eq. (122) reduces to what it is obtained from a deterministic analysis of the circuit [Eq. (17)]. Thus, this equation constitutes an exact stochastic generalization of the deterministic results that to the best of our knowledge was not obtained before.

In Fig. 11(a), we show the probability distribution for the output charge for different values of the power and input voltages. When there is no voltage bias applied to the gate $\left(V_{\mathrm{dd}}=-V_{\mathrm{ss}}=0\right)$, the distribution is just the equilibrium one. When a bias is applied but there is no input voltage $\left(V_{\mathrm{dd}}=-V_{\mathrm{ss}}=5 V_{T}\right.$ and $\left.V_{\mathrm{in}}=0\right)$, the distribution is stretched out and it ceases to be Gaussian. The application of a small input voltage tilts this distribution to one side, and a further increase of the input voltage generates an approximately Gaussian peak centered around the value (a)

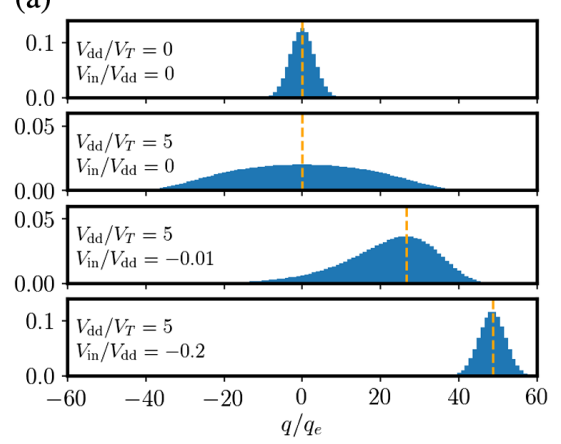

(b)

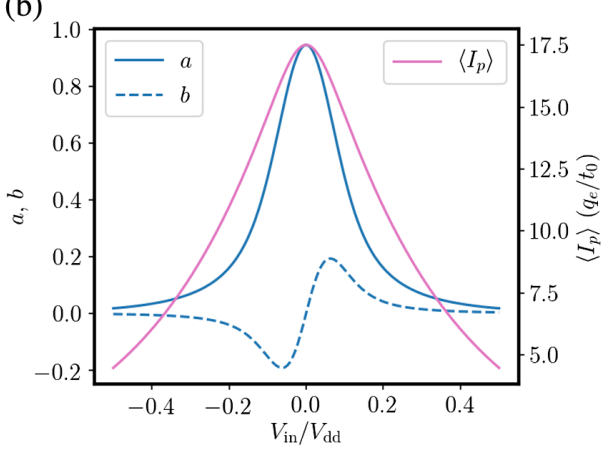

(c)

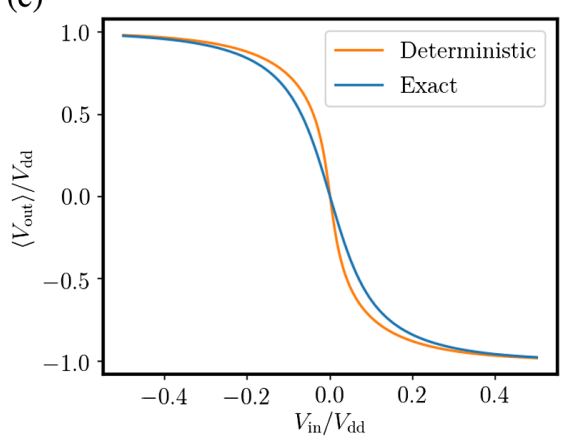

FIG. 11. (a) Probability distributions for the output charge of the inverter for different power and input voltages $\left(q_{e} / q_{T}=0.1\right)$. Here and in the other numerical results, we consider $V_{\mathrm{ss}}=-V_{\mathrm{dd}}$, so that $\Delta V=2 V_{\mathrm{dd}}$. The dashed lines indicate the result of a deterministic analysis. (b) Parameters $a$ and $b$ quantifying the deviations of the fluctuations with respect to thermal equilibrium as a function of $V_{\text {in }}$; see Eq. (123) $\left(V_{\mathrm{dd}} / V_{T}=3\right.$ and $\left.q_{e} / q_{T}=0.5\right)$. We also show the average steady state current through the inverter (in units of $q_{e} / t_{0}$, with $\left.t_{0}=\left(q_{e} / I_{0}\right) \exp \left[V_{\mathrm{th}} /\left(n V_{T}\right)\right]\right)$. (c) Comparison of the transfer function obtained from a deterministic analysis and the exact one taking into account nonequilibrium fluctuations $\left(V_{\mathrm{dd}} / V_{T}=3\right.$ and $\left.q_{e} / q_{T}=0.5\right)$. 
corresponding to the deterministic solution. We see that the distribution of the output charge is, in general, asymmetric with respect to the deterministic value. Thus, its mean value and the deterministic one differ. This result is further evidenced in Fig. 11(b), where we plot the parameters $a$ and $b$ as a function of the input voltage. We see that the largest deviations from equilibrium occur around zero input voltage, when the two transistors are equally activated, while they rapidly decrease as one of the transistors is more activated than the other. What happens here is that for large positive (negative) $V_{\text {in }}$ the output conductor is approximately at equilibrium with the source $V_{\mathrm{ss}}\left(V_{\mathrm{dd}}\right)$. Consequently, the current through the device (and, therefore, the entropy production) follows a similar pattern. Finally, in Fig. 11(c), we show the deviations of the actual transfer function of the inverter from the deterministic one, caused by nonequilibrium fluctuations.

We now turn to the analysis of the current fluctuations. For this analysis, we employ the method for full counting statistics [41,45], that we review in Supplemental Material [109]. This method allows one to evaluate the characteristic function associated to the current fluctuations in terms of the generator of the master equation. Then, the characteristic function can be inverted to obtain the probability distribution. We consider the number $N_{t}$ of charges that got through the pMOS transistor during a time $t$, starting from the stationary distribution. In Fig. 12(a), we show the probability distribution of $N_{t}$ for different input voltages, with $t=10^{-1} t_{0}$, where $t_{0}=\left(q_{e} / I_{0}\right) \exp \left[V_{\text {th }} /\left(n V_{T}\right)\right]$ is the natural timescale for this problem. Also, in Fig. 12(b), we illustrate the DFT in Eq. (106), which in this case reads

$$
\frac{P\left(N_{t}\right)}{P\left(-N_{t}\right)}=e^{N_{t} \Delta V / V_{T}}
$$

assuming an initial state $P_{\text {eq }}(q) \propto e^{-\beta \Psi(q)}$, with $\Psi(q)$ given by Eq. (112).

From Figs. 11(a) and 12(a), we see that, except when the number of charges is too low [as in the bottom in Fig. 12(a)], the deterministic solution matches the most probable result according to the stochastic treatment. This result is analogous to what is formally shown in the case of chemical reaction networks using large deviation theory [92].

These results and methods set the stage for more interesting problems, since the NOT gate is a basic primitive in electronic design in terms of which more complex devices can be built. For example, connecting the output of the gate back to its input through some conduction channel, we can generate self-sustained oscillations. Connecting two NOT gates in a loop, we obtain a bistable system with two metastable NESSs, which is the basis of many designs of electronic memories and also of the next example. More complex logic gates can be modeled in the same way. In this section we analyzed only the stationary distribution of the inverter for a given input voltage, (a)
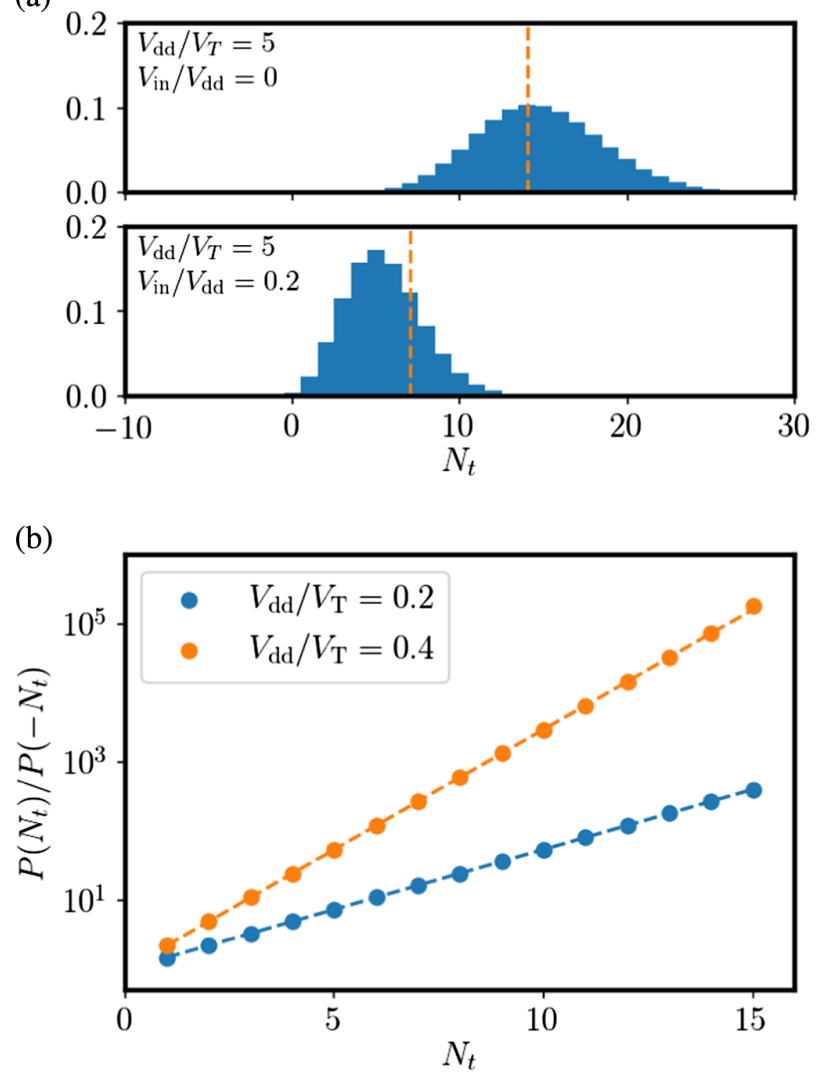

FIG. 12. (a) Probability distributions for the number of charges $N_{t}$ that go through the pMOS transistor during a time $t=10^{-1} t_{0}$ in the stationary state, for different input voltages $\left(q_{e} / q_{T}=0.1\right)$. Dashed lines indicate the values obtained from a deterministic analysis. (b) $P\left(N_{t}\right) / P\left(-N_{t}\right)$ ratio as a function of $N_{t}$ for two different power voltages $\left(q_{e} / q_{T}=0.01\right)$. The dots correspond to the numerical results obtained with the method of full counting statistics, and the dashed lines to the expected result according to the DFT in Eq. (124).

although in a real application the energetic cost of switching the inputs is a significant contribution to the total entropy production. This cost can be analyzed by letting the input change in time in a predefined way. Alternatively, it can also be studied in autonomous circuits (i.e., not requiring time-dependent external driving) displaying bistability or limit cycles, as is done in the next section.

\section{B. A full-CMOS probabilistic bit}

A probabilistic bit ( $p$-bit), or binary stochastic neuron, is a device with a single output $b$ that can ideally take only two values, let us say 1 and -1 . It outputs the value 1 with probability $p$ and -1 with probability $1-p$. The value of $p$ is controlled by an input $I$. For large positive values of $I, p \rightarrow 1$, while for large negative values $p \rightarrow 0$. In a collection of $N$ of these elementary devices, the inputs $\left\{I_{i}\right\}_{i=1, \ldots, N}$ could be adjusted as a function of the instantaneous state $B=\left(b_{1}, b_{2}, \ldots, b_{N}\right)$, and in this way 
correlations between different $p$-bits can be established [14]. For example, given a cost function $E(B)$, it is possible to derive functional relations $I_{i}(B)$ such that the state $B$ occurs with probability $P(B) \propto e^{-E(B)}$. The function $E$ can then be chosen so that its minimum (the most probable state) encodes the solution to some problem of interest [112].

There have been recent proposals to implement $p$-bits with noisy electronic circuits. The most relevant employs magnetic tunnel junctions (MTJs), a technology being used for some commercial memories [113], modified on purpose so that they are sensitive to thermal noise (by lowering the energy barrier separating the two possible states representing one bit) $[13,14]$. A previous proposal considers a "probabilistic switch" [11,114]: a regular CMOS inverter which is driven by external noise at its input, so that its output fluctuates between the two possible values. The advantage of this second proposal is that it is based on CMOS circuits only (not on less common devices like MTJs). However, its main drawback is that the intrinsic noise is actually neglected instead of being exploited as a resource. The reason is that the description of the CMOS inverter is purely deterministic, and, therefore, access to an external source of noise is assumed. Also, the noise is considered to be Gaussian, which as we see in the last section is not always the case. These limitations are, of course, related to the difficulty of describing intrinsic noise in nonlinear electronic circuits, as discussed in Sec. I. As we see next, our formalism allows one to overcome those limitations, which highlights its practical value.

We propose a full-CMOS design for a $p$-bit that is selfsufficient: Its stochastic behavior is due to the intrinsic thermal noise, so no external source of noise is necessary. Also, while in $p$-bits based on MTJs the transition rate or error probability is fixed by the fabrication process (for a given temperature), our design allows one to control this parameter on the fly by just changing the power voltage. The basic circuit is shown in Fig. 13 and is composed of two coupled NOT gates as in regular SRAM cells. The logical circuit in Fig. 13(a) has two stable states: $b=1$ and $\bar{b}=-1$, or $b=-1$ and $\bar{b}=1$. The corresponding CMOS

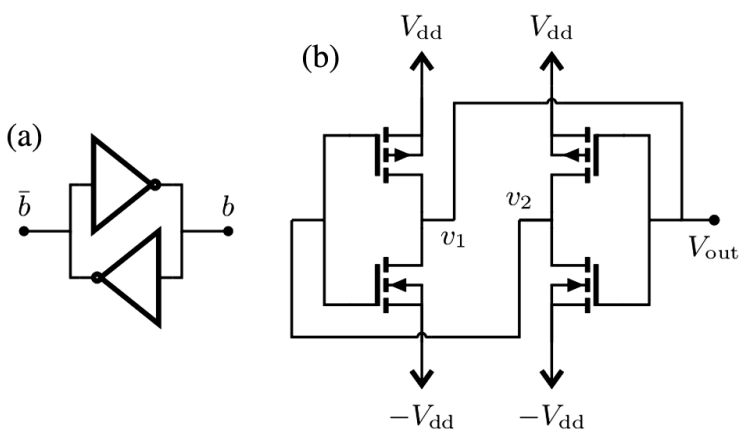

implementation in Fig. 13(b) has two degrees of freedom: the voltages $v_{1}$ and $v_{2}$ (or, alternatively, the charges $q_{1}$ and $q_{2}$ ) at the output of each inverter. We consider $V_{\text {out }}=v_{1}$ to be the output used to monitor the state of the bit. If the powering voltage is above a critical value $V_{\mathrm{dd}}^{*}$ [that for $n=1$ can be found to be $V_{\mathrm{dd}}^{*}=V_{T} \ln (2)$ [90] ], then the deterministic equations for the circuit have two possible steady solutions, which correspond to the two stable logical states in Fig. 13(a). At the stochastic level, they correspond to two metastable NESSs, for which $V_{\text {out }} \simeq V_{\mathrm{dd}}$ or $V_{\text {out }} \simeq-V_{\text {dd }}$, respectively.

A stochastic model for the circuit in Fig. 13(b) can be built as before, by employing the mapping in Fig. 8 and constructing the rates associated to each transistor with the procedure in Sec. IV C. In this case, this construction is done automatically by a custom software package, that is also able to deal with general circuits [115]. The steady state distribution can be obtained by constructing the generator of the master equation in Eq. (12) (truncated to some maximum number of charges) and computing its eigenvector of zero eigenvalue, as shown in Fig. 14(a). Also, the corresponding stochastic dynamics can be simulated with the Gillespie algorithm. In this way, we can generate stochastic trajectories. For example, in Fig. 14(b), we show two trajectories for different values of the power voltage. To obtain those results, we consider the following parameters: $V_{T}=26 \mathrm{mV}$ (room temperature), $C_{g}=50 \mathrm{aF}$, and $C_{o}=10^{-2} C_{g}$. Crucially, these values of capacitances are compatible to what is achieved in modern sub-7-nm fabrication processes [101]. Also, for simplicity, we take $n=1$, and, as before, the parameters $I_{0}$ and $V_{\text {th }}$ of the transistors just fix the timescale $t_{0}=\left(q_{e} / I_{0}\right) \exp \left[V_{\mathrm{th}} /\left(n V_{T}\right)\right]$. We clearly observe random transitions, or errors, between the two metastable NESSs and that the transition or error rate depends on the power voltage $V_{\mathrm{dd}}$. This result is easily understood: Frequent random transitions are expected whenever the standard deviation of the fluctuations around the output voltage, which can be estimated as $\sigma_{V}=\sqrt{k_{b} T /\left[2\left(C_{o}+C_{g}\right)\right]}$, is comparable to the mean value $\left\langle V_{\text {out }}\right\rangle \simeq \pm V_{\text {dd }}$. Thus, one can control the transition rate by changing the powering

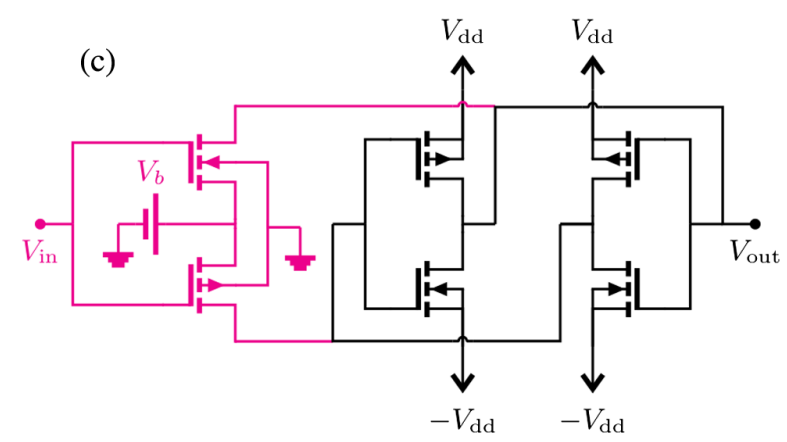

FIG. 13. (a) A bistable circuit constructed with two NOT gates, representing a bit. (b) Its CMOS implementation. (c) Complete design of a $p$-bit. The bistable circuit constituting the bit is shown in black and is the same as in (b). The biasing circuit is shown in magenta. 
(b)

(a)

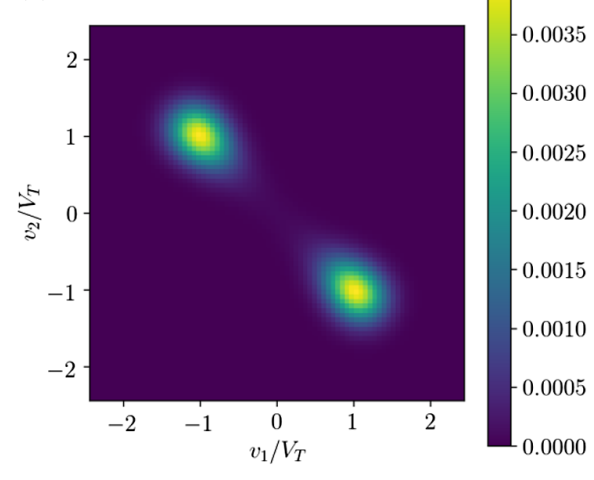

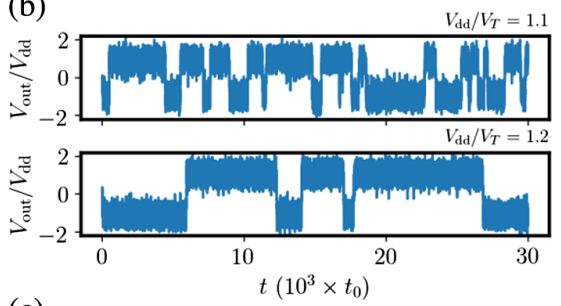

(c)

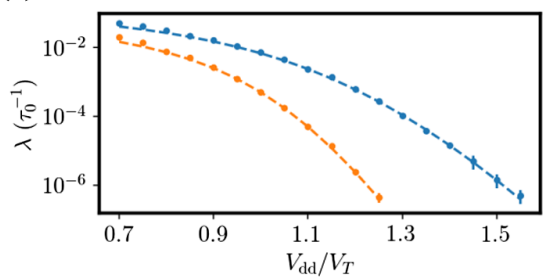

(d)

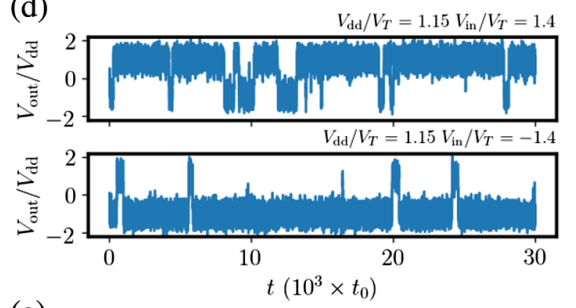

(e)

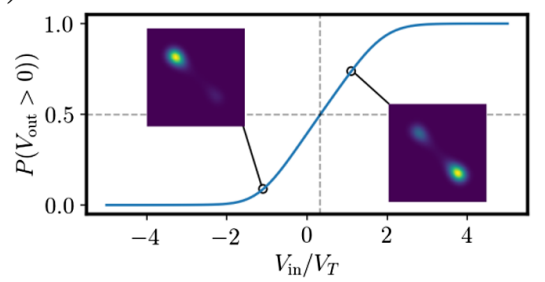

FIG. 14. (a) 2D histogram of the steady state distribution. (b) Output voltage as a function of time for two different power voltages. (c) Transition rate $\lambda$ as a function of the power voltage. Dashed lines correspond to the spectral method developed in Ref. [90], and dots to the calculation of $\lambda$ from stochastic trajectories like the ones in (b). In the last case, the error bars indicate the uncertainty in the determination of $\lambda$ (95\% confidence interval) and can be reduced by generating longer trajectories. The orange line and dots correspond to doubling the scale of the device $\left(C_{o, g} \rightarrow 2 C_{o, g}\right)$. (d) Output voltage as a function of time for two different input voltages $V_{\text {in }}$. (e) Probability of the output being positive as a function of the input voltage. The insets show the steady state distribution for $V_{\text {in }} / V_{T}= \pm 1.1$. In all cases, the parameters are $V_{T}=26 \mathrm{mV}, C_{g}=50 \mathrm{aF}, C_{o}=10^{-2} C_{g}$, and $n=1$.

voltage $V_{\mathrm{dd}}$ or, for fixed $V_{\mathrm{dd}}>V_{\mathrm{dd}}^{*}$, by changing the temperature and or the size of the transistors (which modifies the capacitances $C_{g}$ and $C_{o}$ ). Indeed, to a very good approximation, the waiting time $\tau$ between transitions is exponentially distributed, $P(\tau)=\lambda e^{-\lambda \tau}$, and the transition rate $\lambda$ can be seen to scale as $\lambda \propto$ $\exp \left[-2\left(V_{\mathrm{dd}} / \sigma_{V}\right)^{2} /(n+2)\right]$ to dominant order in $V_{\mathrm{dd}} / \sigma_{V} \gg 1$ [90]. Note that for the previous parameters $V_{\mathrm{dd}}^{*} / \sigma_{V}$ is of the order of one at room temperature but that it increases as the square root of the size of the transistors. Therefore, the transition rate $\lambda$ decreases exponentially in the size of the transistors. As a consequence, the exploitation of these naturally occurring fluctuations as a resource at room temperature is a real possibility only for highly scaled, state of the art fabrication processes, as the ones considered above. The error rate $\lambda$ can be computed from the trajectories generated by the Gillespie algorithm or also by more efficient spectral methods as explained in Ref. [90]. The results are shown in Fig. 14(c) for two different scales.

To complete the construction of the $p$-bit, it is necessary to provide a mechanism to bias its output. There are different ways to achieve this construction, and here we focus on the circuit in Fig. 13(c), where the biasing circuit is colored. It works as follows. A bias voltage $V_{b}>0$ is coupled to the outputs of the two inverters which form the core of the $p$-bit through the drain-source channel of two transistors. The transistor influencing the output of the first inverter is an nMOS, while the one influencing the output of the second inverter is a pMOS. Both transistors have their bodies grounded, such that their activation depends only on the gate-body voltage $V_{\text {in }}$ (the Poisson rates corresponding to this configuration are discussed in Supplemental Material [109]). For $V_{\text {in }}=0$, both transistors are equally activated, and the output of both inverters is very weakly biased toward $V_{b}$. For $V_{\text {in }}>0$, conduction through the nMOS is enhanced, while it is suppressed for the pMOS, and, therefore, only the output of the first inverter is biased toward $V_{b}$. In that case, the symmetry between the two possible metastable NESSs $\left(V_{\text {out }} \simeq V_{\mathrm{dd}}\right.$ or $\left.V_{\text {out }} \simeq-V_{\mathrm{dd}}\right)$ is broken in favor of the one with $V_{\text {out }} \simeq V_{\mathrm{dd}}$. The situation is reversed for $V_{\text {in }}<0$. In Fig. 14(d), we show two sample trajectories of the output voltage $V_{\text {out }}$ for a positive and a negative value of the input voltage $V_{\text {in }}$. We see that $V_{\text {out }}$ is indeed biased and spends more time around positive or negative values, respectively. The parameters of the transistors are the same as before, with the exception that the specific current $I_{0}^{\prime}$ of the transistors in the biasing circuit is one order of magnitude lower than the others $\left(I_{0}^{\prime}=I_{0} / 10\right)$. Also, we consider a bias voltage $V_{b}=V_{T}$. In Fig. 14(e), we show how the probability $p=P\left(V_{\text {out }}>0\right)$ of the output being positive depends on the input voltage. We see that $p$ is indeed given by a sigmoidal function of $V_{\text {in }}$, similar to the typical activation functions considered in artificial neural networks. Note, however, that, due to the asymmetric $I-V$ curves of the transistors in the biasing circuit, the balanced case $P\left(V_{\text {out }}>0\right)=1 / 2$ is not achieved at $V_{\text {in }}=0$ but for a slightly positive $V_{\text {in }}$.

We now analyze the energy consumption of the $p$-bit. For this analysis, we obtain the steady state distribution as before and compute the mean values of current and heat rates associated with each transistor according to Eqs. (57) and (61), respectively. By symmetry, for the circuit constituting the core of the bit in Fig. 13(b), the steady state 
(a)

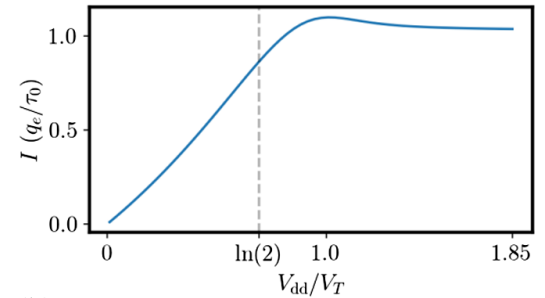

(b)

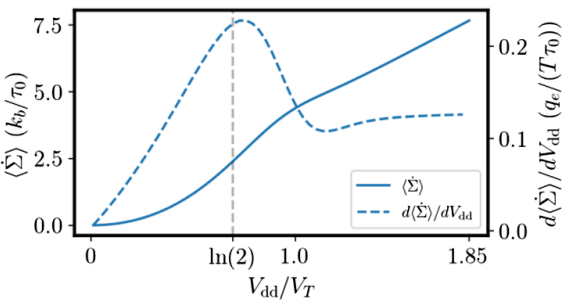

(c)

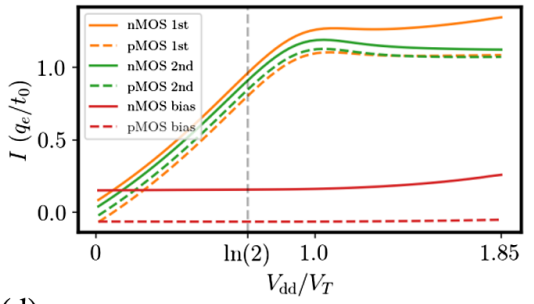

(d)

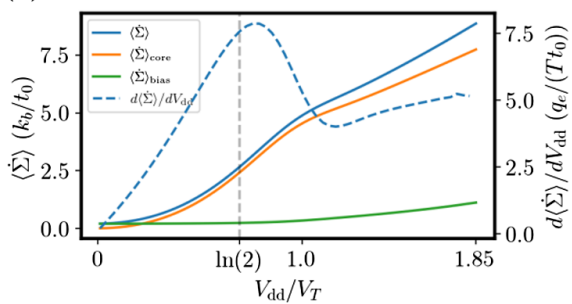

(e)

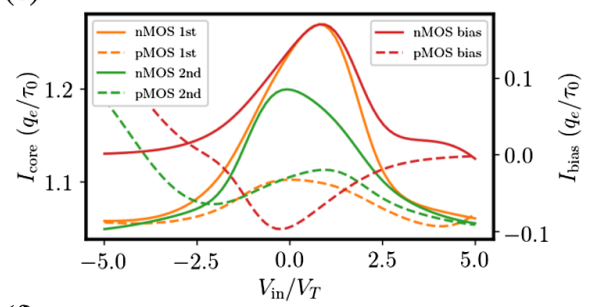

(f)

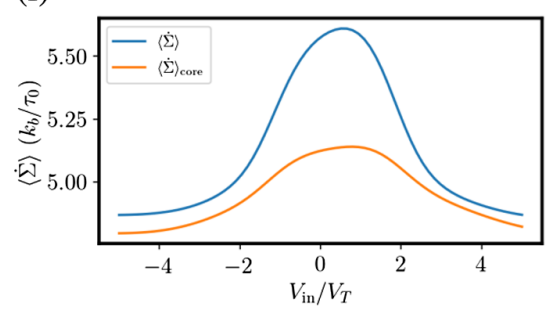

FIG. 15. For the core circuit in Fig. 13(b), we show (a) the average electric current (which is the same for all four transistors) and (b) the total entropy production rate and its derivative with respect to the powering voltage. For the full circuit in Fig. 13(c) with $V_{b}=V_{T}$ and $V_{\text {in }}=0$, we show (c) the average electric current through all the transistors and (d) the total entropy production rate and its derivative. In this case, we also split the contributions to the entropy production associated with the transistors of the core and biasing circuits. In (e) and (f), we show, respectively, the average current through all the transistors and the entropy production rate for the full circuit, this time as a function of the input voltage for $V_{\mathrm{dd}} / V_{T}=1.15$ and $V_{b} / V_{T}=1$. The other parameters are $V_{T}=26 \mathrm{mV}$, $C_{g}=50 \mathrm{aF}, C_{o}=10^{-2} C_{g}$, and $n=1$.

electric current through all four transistors is the same, and its dependence on $V_{\text {dd }}$ is shown in Fig. 15(a). We see that it increases monotonically for low powering voltages and that it develops a peak right after the transition into bistability [at $V_{\mathrm{dd}}=V_{T} \log (2)$ ], after which it settles to a constant value. This maximum in the current close to the transition into bistability is associated to the occurrence of errors or transitions between different NESSs: Each switching event involves the relaxation of previously stored charge, which adds up to the continuous flow of charge in each NESS. This result is also seen in Fig. 15(b), where we plot the entropy production rate and its derivative with respect to the powering voltage. Since we are considering steady state conditions, we have that $\langle\dot{\Sigma}\rangle=\left\langle\dot{\Sigma}_{e}\right\rangle=(-1 / T)\left(\left\langle\dot{Q}_{p 1}\right\rangle+\right.$ $\left.\left\langle\dot{Q}_{n 1}\right\rangle+\left\langle\dot{Q}_{p 2}\right\rangle+\left\langle\dot{Q}_{n 2}\right\rangle\right)$, where $\left\langle\dot{Q}_{(n / p)(1 / 2)}\right\rangle$ is the rate of heat dissipation in the nMOS or pMOS transistor of the first or second inverter. Interestingly, we see that the transition to bistability is signaled by a maximum in the derivative of $\langle\dot{\Sigma}\rangle$ with respect to $V_{\text {dd }}$.

In Fig. 15(c), we show all the currents for the full circuit in Fig. 13(c), including the bias circuit, for $V_{b} / V_{T}=1$ and $V_{\text {in }}=0$. We see that the behavior of the currents through the transistor in the core circuit is qualitatively similar as the previous case, although the currents are naturally not all equal anymore. In Fig. 15(d), we show the total entropy production and its derivative for the full circuit. In this case, we split the total entropy production rate as $\langle\dot{\Sigma}\rangle=\langle\dot{\Sigma}\rangle_{\text {core }}+\langle\dot{\Sigma}\rangle_{\text {bias }}$, where $\langle\dot{\Sigma}\rangle_{\text {core }}=(-1 / T)\left(\left\langle\dot{Q}_{p 1}\right\rangle+\right.$ $\left.\left\langle\dot{Q}_{n 1}\right\rangle+\left\langle\dot{Q}_{p 2}\right\rangle+\left\langle\dot{Q}_{n 2}\right\rangle\right)$ and $\langle\dot{\Sigma}\rangle_{\text {bias }}=(-1 / T)\left(\left\langle\dot{Q}_{p b}\right\rangle+\right.$ $\left.\left\langle\dot{Q}_{n b}\right\rangle\right)$ are the rate entropy production rates corresponding to the bit core and biasing circuits $\left(\left\langle\dot{Q}_{(n / p) b}\right\rangle\right.$ is the rate of heat dissipation in the nMOS or pMOS biasing transistor). We see that the transition to bistability is still signaled by a maximum in the derivative of $\langle\dot{\Sigma}\rangle$ and that the dissipation associated to the biasing circuit is a small fraction of the total one.

Finally, the behavior with respect to the input voltage $V_{\text {in }}$ with fixed powering voltage $V_{\mathrm{dd}} / V_{T}=1.15$ and bias voltage $V_{b} / V_{T}=1$ is shown in Figs. 15(e) and 15(f). In Fig. 15(f), we see that the entropy production rate has a maximum value at zero bias. This result is again due to the occurrence of transitions between different NESSs, which, of course, decrease when the bias increases in any direction.

The average total dissipated heat per generated bit is given by $\bar{Q}=T\langle\dot{\Sigma}\rangle / \lambda$. It is interesting to note that, for transitions generated by intrinsic thermal noise, $\bar{Q}$ decreases as the speed or transition rate increases. For example, by reducing the powering voltage $V_{\mathrm{dd}}$ (always above $V_{\mathrm{dd}}^{*}$ ), the total rate of heat dissipation $T\langle\dot{\Sigma}\rangle$ decreases [Figs. 15(b) and 15(d)], while the transition rate $\lambda$ increases exponentially [Fig. 14(c)], and, therefore, $\bar{Q}$ decreases exponentially. For $V_{\mathrm{dd}} / V_{T} \simeq 1.1$, we have an average dissipated heat per generated bit on the order of $\bar{Q}=T\langle\dot{\Sigma}\rangle / \lambda \simeq 2 \times 10^{3} k_{b} T \simeq 10$ aJ. This result can be compared to the MTJ $p$-bit in Ref. [14] that requires an energy of $2 \mathrm{fJ}$ per random bit, 2 orders of magnitude higher than the previous estimation.

\section{DISCUSSION}

We have presented a formalism for the construction of stochastic models of nonlinear electronic circuits in a 
thermodynamically consistent way. Devices with arbitrary $I-V$ curves can be described, provided that their current fluctuations display shot noise. A complete analysis of the stochastic thermodynamics of these models was carried out. The relevant thermodynamic potentials were identified, and the different contributions to the irreversible entropy production were characterized. All these quantities were extended to individual trajectories, based on which we presented different detailed and integral fluctuation theorems. As a first application, we have constructed a stochastic model of a subthreshold CMOS inverter, or NOT gate. We have shown how to analytically find the steady state of the resulting master equation. Based on that solution, we analyzed how the nonequilibrium thermal fluctuations induce modifications in the transfer function of the gate. Also, we showed how to compute the full counting statistics of the current fluctuations and in that way illustrated a detailed fluctuation theorem. Finally, we proposed a fullCMOS design of a probabilistic bit, or binary stochastic neuron, in which intrinsic thermal noise is exploited as a resource to generate random bits of information in a controllable way. The energy consumption of our design is several times lower than in previous proposals.

Of course, the formalism has some limitations, which are important to discuss here. In first place, only devices displaying shot noise can be described. This limitation excludes, for example, regular resistors or MOS transistors in general modes of operation. In addition, it is not possible to describe inertial effects (i.e., inductances). These could be included at the price of mixing discrete and continuous variations of charge. Nevertheless, there is little practical motivation for this inclusion, since it is difficult to integrate inductances in nanoscale electronic circuits [116], and, therefore, inertial effects might be relevant only at extremely high frequencies. There are other possible extensions of the formalism, which are, however, less relevant, since they capture effects that can be actually emulated with the formalism as presented here. For example, although the stochastic dynamics we have considered is Markovian, non-Markovian effects can be described by considering a given circuit as a part of a larger one (something known as "Markovian embedding"). Finally, although we have not focused on single-electron devices, our formalism can be directly applied to them. In that context, there are "cotunneling" effects (events in which two or more transitions happen at the same time, possibly leaving the state of the circuit unchanged) that can become relevant in the Coulomb blockade regime and which our formalism does not take into account [40].

Our work bridges between different subjects and communities. It shows how to employ the methods of stochastic thermodynamics and single-electron devices to model other kinds of circuits that are traditionally first described deterministically and then supplemented by an approximate treatment of the noise. In this way, we can describe the fluctuations in those circuits on a rigorous basis. This description is relevant and timely in view of the impressive reduction in the size of CMOS circuits and the need for new energy-efficient computing paradigms. On another front, the great versatility in the fabrication and control of electronic circuits makes them an excellent platform to study complex phenomena in statistical physics and nonequilibrium thermodynamics. Thus, our formalism also offers a valuable bridge between theory and experiment. In the future, it could be interesting to explore the connection between the low-level description we propose here and the more abstract treatments of stochastic thermodynamics of complex circuits in Refs. [74,75], in particular, to identify under which conditions the fundamental bounds that they obtained can be approached within a given technology.

After finishing this work, we became aware of a recent article [9], in which a similar stochastic description is employed to compute the error rate of a low-power SRAM memory cell. It should be noted that the transition rates considered in that article are actually not thermodynamically consistent; i.e., they do not respect the local detailed balance conditions. Also, after submission of our work, a related paper appeared [117], using ST to model logical operations using single-electron devices.

\section{ACKNOWLEDGMENTS}

J.-C. D. thanks Denis Flandre and Lopold Van Brandt for helpful comments and discussions. The authors acknowledge funding from the INTER project "TheCirco" (INTER/FNRS/20/15074473), funded by the F.R.S.-FNRS (Belgium) and FNR (Luxembourg). N. F. and M. E. also acknowledge funding from the European Research Council, project NanoThermo (ERC-2015-CoG Agreement No. 681456), and from the FNR CORE program, project NTEC (C19/MS/13664907). J.-C. D. was also funded by the FNR Program No. INTER/MOBILITY/18/12987626.

[1] C. Mora, R. L. Rollins, K. Taladay, M. B. Kantar, M. K. Chock, M. Shimada, and E. C. Franklin, Bitcoin Emissions Alone Could Push Global Warming above 2 C, Nat. Clim. Change 8, 931 (2018).

[2] E. Masanet, A. Shehabi, N. Lei, S. Smith, and J. Koomey, Recalibrating Global Data Center Energy-Use Estimates, Science 367, 984 (2020).

[3] L. B. Kish, End of Moore's Law: Thermal (Noise) Death of Integration in Micro and Nano Electronics, Phys. Lett. A 305, 144 (2002).

[4] A. S. Sadek, K. Nikolić, and M. Forshaw, Parallel Information and Computation with Restitution for NoiseTolerant Nanoscale Logic Networks, Nanotechnology 15, 192 (2003).

[5] S. Krishnan, S. V. Garimella, G. M. Chrysler, and R. V. Mahajan, Towards a Thermal Moore's Law, IEEE Trans. Adv. Packag. 30, 462 (2007). 
[6] L. B. Kish, Noise-Based Logic: Binary, Multi-valued, or Fuzzy, with Optional Superposition of Logic States, Phys. Lett. A 373, 911 (2009).

[7] T. J. Hamilton, S. Afshar, A. van Schaik, and J. Tapson, Stochastic Electronics: A Neuro-Inspired Design Paradigm for Integrated Circuits, Proc. IEEE 102, 843 (2014).

[8] A. O. Orlov, I. K. Hänninen, C. O. Campos-Aguillón, R. Celis-Cordova, M. S. McConnell, G. P. Szakmany, C. C. Thorpe, B.T. Appleton, G. P. Boechler, C. S. Lent et al., Experimental Tests of the Landauer Principle in Electron Circuits, and Quasi-adiabatic Computing Systems, in Energy Limits in Computation (Springer, New York, 2019), pp. 177-230.

[9] E. Rezaei, M. Donato, W. R. Patterson, A. Zaslavsky, and R. I. Bahar, Fundamental Thermal Limits on Data Retention in Low-Voltage CMOS Latches and SRAM, IEEE Trans. Device Mater. Reliab. 20, 488 (2020).

[10] K. V. Palem, Energy Aware Computing through Probabilistic Switching: A Study of Limits, IEEE Trans. Comput. 54, 1123 (2005).

[11] S. Cheemalavagu, P. Korkmaz, K. V. Palem, B. E. S. Akgul, and L. N. Chakrapani, A Probabilistic CMOS Switch and Its Realization by Exploiting Noise, on Proceedings of the IFIP International Conference on VLSI (CiteSeer, 2005), pp. 535-541.

[12] J. Han and M. Orshansky, Approximate Computing: An Emerging Paradigm for Energy-Efficient Design, in Proceedings of the 2013 18th IEEE European Test Symposium (ETS) (IEEE, New York, 2013), pp. 1-6.

[13] K. Y. Camsari, R. Faria, B. M. Sutton, and S. Datta, Stochastic p-Bits for Invertible Logic, Phys. Rev. X 7 , 031014 (2017).

[14] W. A. Borders, A. Z. Pervaiz, S. Fukami, K. Y. Camsari, H. Ohno, and S. Datta, Integer Factorization Using Stochastic Magnetic Tunnel Junctions, Nature (London) 573, 390 (2019).

[15] J. Kaiser, R. Faria, K. Y. Camsari, and S. Datta, Probabilistic Circuits for Autonomous Learning: A Simulation Study, Front. Comput. Neurosci. 14, 14 (2020).

[16] Y. M. Blanter and M. Büttiker, Shot Noise in Mesoscopic Conductors, Phys. Rep. 336, 1 (2000).

[17] R. McFee, Self-Rectification in Diodes and the Second Law of Thermodynamics, Am. J. Phys. 39, 814 (1971).

[18] N. G. Van Kampen, Non-linear Thermal Fluctuations in a Diode, Physica (Utrecht) 26, 585 (1960).

[19] L. Weiss and W. Mathis, A Thermodynamical Approach to Noise in Non-linear Networks, Int. J. Circuit Theory Appl. 26, 147 (1998).

[20] J. L. Wyatt, Jr. and G. J. Coram, Nonlinear Device Noise Models: Thermodynamic Requirements, technical report, 1997.

[21] J. L. Wyatt and G. J. Coram, Nonlinear Device Noise Models: Satisfying the Thermodynamic Requirements, IEEE Trans. Electron Devices 46, 184 (1999).

[22] L. Brillouin, Can the Rectifier Become a Thermodynamical Demon?, Phys. Rev. 78, 627 (1950).

[23] H.-N. Tan and J. Wyatt, Thermodynamics of Electrical Noise in a Class of Nonlinear RLC Networks, IEEE Trans. Circ. Syst. 32, 540 (1985).
[24] M. Esposito, U. Harbola, and S. Mukamel, Nonequilibrium Fluctuations, Fluctuation Theorems, and Counting Statistics in Quantum Systems, Rev. Mod. Phys. 81, 1665 (2009).

[25] M. Campisi, P. Hänggi, and P. Talkner, Colloquium: Quantum Fluctuation Relations: Foundations and Applications, Rev. Mod. Phys. 83, 771 (2011).

[26] C. Jarzynski, Equalities and Inequalities: Irreversibility and the Second Law of Thermodynamics at the Nanoscale, Annu. Rev. Condens. Matter Phys. 2, 329 (2011).

[27] U. Seifert, Stochastic Thermodynamics, Fluctuation Theorems and Molecular Machines, Rep. Prog. Phys. 75, 126001 (2012).

[28] R. Rao and M. Esposito, Detailed Fluctuation Theorems: A Unifying Perspective, Entropy 20, 635 (2018).

[29] K. Sekimoto, Stochastic Energetics (Springer, New York, 2010), Vol. 799.

[30] X.-J. Zhang, H. Qian, and M. Qian, Stochastic Theory of Nonequilibrium Steady States and Its Applications. Part I, Phys. Rep. 510, 1 (2012).

[31] R. Rao and M. Esposito, Conservation Laws Shape Dissipation, New J. Phys. 20, 023007 (2018).

[32] S. Ciliberto, Experiments in Stochastic Thermodynamics: Short History and Perspectives, Phys. Rev. X 7, 021051 (2017).

[33] R. Rao and M. Esposito, Conservation Laws and Work Fluctuation Relations in Chemical Reaction Networks, J. Chem. Phys. 149, 245101 (2018).

[34] J. M. R. Parrondo and B. J. de Cisneros, Energetics of Brownian Motors: A Review, Appl. Phys. A 75, 179 (2002).

[35] R. Van Zon, S. Ciliberto, and E. G. D. Cohen, Power and Heat Fluctuation Theorems for Electric Circuits, Phys. Rev. Lett. 92, 130601 (2004).

[36] N. Garnier and S. Ciliberto, Nonequilibrium Fluctuations in a Resistor, Phys. Rev. E 71, 060101(R) (2005).

[37] S. Ciliberto, A. Imparato, A. Naert, and M. Tanase, Heat Flux and Entropy Produced by Thermal Fluctuations, Phys. Rev. Lett. 110, 180601 (2013).

[38] N. Freitas, J.-C. Delvenne, and M. Esposito, Stochastic and Quantum Thermodynamics of Driven RLC Networks, Phys. Rev. X 10, 031005 (2020).

[39] M. H. Devoret, D. Estève, H. Grabert, G.-L. Ingold, H. Pothier, and C. Urbina, Effect of the Electromagnetic Environment on the Coulomb Blockade in Ultrasmall Tunnel Junctions, Phys. Rev. Lett. 64, 1824 (1990).

[40] C. Wasshuber, Computational Single-Electronics (Springer Science, New York, 2012).

[41] D. A. Bagrets and Yu. V. Nazarov, Full Counting Statistics of Charge Transfer in Coulomb Blockade Systems, Phys. Rev. B 67, 085316 (2003).

[42] D. V. Averin and K. K. Likharev, Coulomb Blockade of Single-Electron Tunneling, and Coherent Oscillations in Small Tunnel Junctions, J. Low Temp. Phys. 62, 345 (1986).

[43] D. Ali and H. Ahmed, Coulomb Blockade in a Silicon Tunnel Junction Device, Appl. Phys. Lett. 64, 2119 (1994).

[44] D. Andrieux and P. Gaspard, Fluctuation Theorem for Transport in Mesoscopic Systems, J. Stat. Mech. (2006) P01011.

[45] M. Esposito, U. Harbola, and S. Mukamel, Fluctuation Theorem for Counting Statistics in Electron Transport 
through Quantum Junctions, Phys. Rev. B 75, 155316 (2007).

[46] G. B. Cuetara, M. Esposito, and P. Gaspard, Fluctuation Theorems for Capacitively Coupled Electronic Currents, Phys. Rev. B 84, 165114 (2011).

[47] J. V. Koski, T. Sagawa, O. P. Saira, Y. Yoon, A. Kutvonen, P. Solinas, M. Möttönen, T. Ala-Nissila, and J. P. Pekola, Distribution of Entropy Production in a Single-Electron Box, Nat. Phys. 9, 644 (2013).

[48] G. B. Cuetara and M. Esposito, Double Quantum Dot Coupled to a Quantum Point Contact: A Stochastic Thermodynamics Approach, New J. Phys. 17, 095005 (2015).

[49] J. P. Pekola, Towards Quantum Thermodynamics in Electronic Circuits, Nat. Phys. 11, 118 (2015).

[50] G. Benenti, G. Casati, K. Saito, and R. S. Whitney, Fundamental Aspects of Steady-State Conversion of Heat to Work at the Nanoscale, Phys. Rep. 694, 1 (2017).

[51] J. Gu and P. Gaspard, Microreversibility, Fluctuations, and Nonlinear Transport in Transistors, Phys. Rev. E 99, 012137 (2019).

[52] I. Amlani, A. O. Orlov, G. Toth, G. H. Bernstein, C. S. Lent, and G. L. Snider, Digital Logic Gate Using Quantum-Dot Cellular Automata, Science 284, 289 (1999).

[53] C. C. Enz and E. A. Vittoz, Charge-Based MOS Transistor Modeling (Wiley, New York, 2006).

[54] Y. Tsividis and C. McAndrew, Operation and Modeling of the MOS Transistor (Oxford University, New York, 2011).

[55] R. Sarpeshkar, T. Delbruck, and C. A. Mead, White Noise in MOS Transistors and Resistors, IEEE Circuits and Devices Magazine 9, 23 (1993).

[56] J. Gu and P. Gaspard, Stochastic Approach and Fluctuation Theorem for Charge Transport in Diodes, Phys. Rev. E 97, 052138 (2018).

[57] J. Gu and P. Gaspard, Counting Statistics and Microreversibility in Stochastic Models of Transistors, J. Stat. Mech. (2020) 103206.

[58] D. H. Wolpert, The Stochastic Thermodynamics of Computation, J. Phys. A 52, 193001 (2019).

[59] T. Sagawa, Second Law, Entropy Production, and Reversibility in Thermodynamics of Information, in Energy Limits in Computation (Springer, New York, 2019), pp. 101-139.

[60] C. H. Bennett, The Thermodynamics of Computation Review, Int. J. Theor. Phys. 21, 905 (1982).

[61] C. H. Bennett and R. Landauer, The Fundamental Physical Limits of Computation, Sci. Am. 253, 48 (1985).

[62] T. Sagawa, Thermodynamic and Logical Reversibilities Revisited, J. Stat. Mech. (2014) P03025.

[63] T. Sagawa and M. Ueda, Minimal Energy Cost for Thermodynamic Information Processing: Measurement and Information Erasure, Phys. Rev. Lett. 102, 250602 (2009).

[64] J. M. Horowitz and M. Esposito, Thermodynamics with Continuous Information Flow, Phys. Rev. X 4, 031015 (2014).

[65] K. Ptaszyński and M. Esposito, Thermodynamics of Quantum Information Flows, Phys. Rev. Lett. 122, 150603 (2019).

[66] E. Aurell, K. Gawedzki, C. Mejia-Monasterio, R. Mohayaee, and P. Muratore-Ginanneschi, Refined Second Law of
Thermodynamics for Fast Random Processes, J. Stat. Phys. 147, 487 (2012).

[67] T. Schmiedl and U. Seifert, Optimal Finite-Time Processes in Stochastic Thermodynamics, Phys. Rev. Lett. 98, 108301 (2007).

[68] D. A. Sivak and G. E. Crooks, Thermodynamic Metrics and Optimal Paths, Phys. Rev. Lett. 108, 190602 (2012).

[69] S. B. Nicholson, L. Pedro Garcia-Pintos, A. del Campo, and J. R. Green, Time-Information Uncertainty Relations in Thermodynamics, Nat. Phys. 16, 1211 (2020).

[70] G. Diana, G. B. Bagci, and M. Esposito, Finite-Time Erasing of Information Stored in Fermionic Bits, Phys. Rev. E 87, 012111 (2013).

[71] P. R. Zulkowski and M. R. DeWeese, Optimal Finite-Time Erasure of a Classical Bit, Phys. Rev. E 89, 052140 (2014).

[72] K. Proesmans, J. Ehrich, and J. Bechhoefer, Finite-Time Landauer Principle, Phys. Rev. Lett. 125, 100602 (2020).

[73] S. Sheng, T. Herpich, G. Diana, and M. Esposito, Thermodynamics of Majority-Logic Decoding in Information Erasure, Entropy 21, 284 (2019).

[74] A. B. Boyd, D. Mandal, and J. P. Crutchfield, Thermodynamics of Modularity: Structural Costs beyond the Landauer Bound, Phys. Rev. X 8, 031036 (2018).

[75] D. Wolpert and A. Kolchinsky, The Thermodynamics of Computing with Circuits, New J. Phys. 22, 063047 (2020).

[76] A. Wang, B. H. Calhoun, and A.P. Chandrakasan, Sub-threshold Design for Ultra Low-Power Systems (Springer, New York, 2006), Vol. 95.

[77] J.-W. Han, J. S. Oh, and M. Meyyappan, Vacuum Nanoelectronics: Back to the Future?-Gate Insulated Nanoscale Vacuum Channel Transistor, Appl. Phys. Lett. 100, 213505 (2012).

[78] D. H. Ackley, G. E. Hinton, and T. J. Sejnowski, A Learning Algorithm for Boltzmann Machines, Cogn. Sci. 9, 147 (1985).

[79] Y. Bengio, N. Léonard, and A. Courville, Estimating or Propagating Gradients through Stochastic Neurons for Conditional Computation, arXiv:1308.3432.

[80] K. Nawaz, L. Van Brandt, Itamar Levi, François-Xavier Standaert, and Denis Flandre, A Security Oriented Transient-Noise Simulation Methodology: Evaluation of Intrinsic Physical Noise of Cryptographic Designs, Integration, The VLSI Journal 68, 71 (2019).

[81] A. J. Scholten, R. Van Langevelde, L. F. Tiemeijer, and D. B. M. Klaassen, Compact Modeling of Noise in CMOS, in Proceedings of the IEEE Custom Integrated Circuits Conference 2006 (IEEE, New York, 2006), pp. 711-716.

[82] M. Polettini and M. Esposito, Irreversible Thermodynamics of Open Chemical Networks. I. Emergent Cycles and Broken Conservation Laws, J. Chem. Phys. 141, 024117 (2014).

[83] A given circuit with two-terminal devices can be mapped to a chemical reaction network by mapping conductors to chemical species $A, B, C, \ldots$ and devices to chemical reactions $X \leftrightarrow Y$. This mapping concerns only the structure of the circuit and network, not the dynamics or thermodynamics.

[84] L. O. Chua, C. A. Desoer, and E. S. Kuh, Linear and Nonlinear Circuits (McGraw-Hill, New York, 1987). 
[85] P. Hanggi and P. Jung, Bistability in Active Circuits: Application of a Novel Fokker-Planck Approach, IBM J. Res. Dev. 32, 119 (1988).

[86] J. M. Horowitz, Diffusion Approximations to the Chemical Master Equation Only Have a Consistent Stochastic Thermodynamics at Chemical Equilibrium, J. Chem. Phys. 143, 044111 (2015).

[87] M. Doi, Second Quantization Representation for Classical Many-Particle System, J. Phys. A 9, 1465 (1976).

[88] A. Lefevre and G. Biroli, Dynamics of Interacting Particle Systems: Stochastic Process and Field Theory, J. Stat. Mech. (2007) P07024.

[89] H. Touchette, The Large Deviation Approach to Statistical Mechanics, Phys. Rep. 478, 1 (2009).

[90] N. Freitas, K. Proesmans, and M. Esposito, Reliability and Entropy Production in Non-Equilibrium Electronic Memories, arXiv:2103.01184.

[91] N. Freitas, G. Falasco, and M. Esposito, Linear Response in Large Deviations Theory: A Method to Compute Nonequilibrium Distributions, arXiv:2106.05887.

[92] A. Lazarescu, T. Cossetto, G. Falasco, and M. Esposito, Large Deviations and Dynamical Phase Transitions in Stochastic Chemical Networks, J. Chem. Phys. 151, 064117 (2019).

[93] R. Landauer, Solid-State Shot Noise, Phys. Rev. B 47, 16427 (1993).

[94] T. A. Fulton and G. J. Dolan, Observation of SingleElectron Charging Effects in Small Tunnel Junctions, Phys. Rev. Lett. 59, 109 (1987).

[95] W. Shockley, The Theory of $p$-n Junctions in Semiconductors and $p-n$ Junction Transistors, Bell Syst. Tech. J. 28, 435 (1949).

[96] M. J. Kirton and M. J. Uren, Noise in Solid-State Microstructures: A New Perspective on Individual Defects, Interface States and Low-Frequency (1/) Noise, Adv. Phys. 38, 367 (1989).

[97] X. Chen, L. Wang, B. Li, Yu. Wang, X. Li, Y. Liu, and H. Yang, Modeling Random Telegraph Noise as a Randomness Source and Its Application in True Random Number Generation, IEEE Trans. Comput. Aided Des. Integr. Circ. Syst. 35, 1435 (2015).

[98] L. Van Brandt, B. K. Esfeh, V. Kilchytska, and D. Flandre, Robust Methodology for Low-Frequency Noise Power Analyses in Advanced MOS Transistors, in Proceedings of the 2019 Joint International EUROSOI Workshop and International Conference on Ultimate Integration on Silicon (EUROSOI-ULIS) Grenoble, France, (2019), pp. 1-4.

[99] E. Paladino, Y. M. Galperin, G. Falci, and B. L. Altshuler, 1/f Noise: Implications for Solid-State Quantum Information, Rev. Mod. Phys. 86, 361 (2014).

[100] H. Grabert and M. H. Devoret, Single Charge Tunneling: Coulomb Blockade Phenomena in Nanostructures (Springer Science, New York, 2013), Vol. 294.
[101] P. Zheng, Advanced MOSFET Structures and Processes for Sub-7 nm CMOS Technologies, Ph.D. thesis, UC Berkeley, 2016.

[102] T. Cossetto, Problems in Nonequilibrium Fluctuations across Scales: A Path Integral Approach, Dissertation thesis, University of Luxembourg, Luxembourg, Luxembourg, 2020, http://hdl.handle.net/10993/45484.

[103] M. Esposito and C. Van den Broeck, Second Law and Landauer Principle far from Equilibrium, Europhys. Lett. 95, 40004 (2011).

[104] T. Sagawa, Thermodynamics of Information Processing in Small Systems, Prog. Theor. Phys. 127, 1 (2012).

[105] J. M. R. Parrondo, J. M. Horowitz, and T. Sagawa, Thermodynamics of Information, Nat. Phys. 11, 131 (2015).

[106] P. M. Riechers, Transforming Metastable Memories: The Nonequilibrium Thermodynamics of Computation, arXiv: 1808.03429.

[107] T. Speck and U. Seifert, Integral Fluctuation Theorem for the Housekeeping Heat, J. Phys. A 38, L581 (2005).

[108] A. Kolchinsky and D. H. Wolpert, Entropy Production and Thermodynamics of Information under Protocol Constraints, arXiv:2008.10764.

[109] See Supplemental Material at http://link.aps.org/ supplemental/10.1103/PhysRevX.11.031064 for derivations supporting some of the results in the main text.

[110] U. Seifert, Fluctuation Theorem for a Single Enzyme or Molecular Motor, Europhys. Lett. 70, 36 (2005).

[111] A. M. Timpanaro, G. Guarnieri, J. Goold, and G. T. Landi, Thermodynamic Uncertainty Relations from Exchange Fluctuation Theorems, Phys. Rev. Lett. 123, 090604 (2019).

[112] A. Lucas, Ising Formulations of Many NP Problems, Front. Phys. 2, 5 (2014).

[113] S. Bhatti, R. Sbiaa, A. Hirohata, H. Ohno, S. Fukami, and S. N. Piramanayagam, Spintronics Based Random Access Memory: A Review, Mater. Today 20, 530 (2017).

[114] B. E. S. Akgul, L. N. Chakrapani, P. Korkmaz, and K. V. Palem, Probabilistic CMOS Technology: A Survey and Future Directions, in Proceedings of the 2006 IFIP International Conference on Very Large Scale Integration (IEEE, New York, 2006), pp. 1-6.

[115] N. Freitas, Stochastic Single Electronics (SSE), https:// github.com/nfreitas/SSE.

[116] T. M. Philip and M. J. Gilbert, High-Performance Nanoscale Topological Inductor, in Proceedings of the 2017 75th Annual Device Research Conference (DRC) (IEEE, New York, 2017), pp. 1-2.

[117] C. Y. Gao and D. T. Limmer, Principles of Low Dissipation Computing from a Stochastic Circuit Model, arXiv:2102.13067. 